\title{
Metallocalix[4]arene Polymers for Gravimetric Detection of $N$-Nitrosodialkylamines
}

Ru-Qiang Lu, ${ }^{\mathrm{a}}$ Weize Yuan ${ }^{\mathrm{a}}$, Robert G. Croy, ${ }^{\mathrm{b}}$ John M. Essigmann, ${ }^{\mathrm{a}, \mathrm{b}}$ and Timothy M. Swager*a

${ }^{a}$ Department of Chemistry and ${ }^{b}$ Departments of Biological Engineering and Center for Environmental Health Sciences, Massachusetts Institute of Technology, 77 Massachusetts Avenue, Cambridge, Massachusetts 02139, United States

Table of Contents

1. Instruments, methods and chemicals

2. Synthesis

3. Supplementary Figures and Tables

4. Theoretical calculations

5. NMR spectra

6. Reference 


\section{Instruments, methods and chemicals}

Instruments and chemicals. All chemicals were used as received unless otherwise noted. NDMA was prepared following the reported procedures. ${ }^{\mathrm{S} 1}$ NDEA and NDBA were purchased from Sigma-Aldrich and TCI, respectively. Fourier transform-infrared (FT-IR) spectroscopy was performed on a Thermo Scientific Nicolet 6700 Fourier transform infrared spectrometer. Scanning electron microscope (SEM) was performed on the Merlin and Crossbeam 540 Zeiss SEM. High-resolution mass spectrometry was performed on an Agilent Infinity 1260 LC system coupled to a high-resolution Agilent 6545 mass spectrometer. A Jet Stream ESI source was used. Thermogravimetric analysis was recorded on a TA Thermogravimetric Analyzer in nitrogen. NMR spectra were collected on a Bruker Avance $400 \mathrm{MHz}$ or $500 \mathrm{MHz}$ spectrometer. Analyte gases were generated by a FlexStream FlexBase module. The dilute flow was controled by a mass flow controller (MFC). The sensing setups are similar to the previous report (Figure S6). ${ }^{\mathrm{S} 1}$ Gel permeation chromatography (GPC) was recorded on an Agilent 1260 Infinity system with dual Agilent PL1110-6500 columns using a chloroform mobile phase.

QCM measurement. Gravimetric sensing was performed on a Quartz Crystal Microbalance (QCM) QE401F1318. Gold Qsensors were purchased from Nanoscience Instruments (QSX 301). Gold Qsensors can be repeatedly used with proper cleaning. The organic materials on used gold Qsensors were removed by dichloromethane. Then the gold sensors were soaked in the bath of a mixture of Milli-Q ultrapure water, ammonia (aqueous, 28-30\%), and hydrogen peroxide (aqueous, 30\%) $(\mathrm{v} / \mathrm{v} / \mathrm{v}=5 / 1 / 1)$ at $75^{\circ} \mathrm{C}$ for $5 \mathrm{~min}$ with stirring. Clean sensors were rinsed with Milli-Q ultrapure water and dried under a stream of filtered nitrogen. After being dried, sensors were treated with oxygen plasma for 30 seconds. New sensors were cleaned using the same method. The polymer solution in dichloromethane $(3 \mathrm{mg} / \mathrm{mL}, 10 \mu \mathrm{L})$ was dropcast on clean sensors. The sensor with polymer film was dried under vacuum before measurement. The response was defined as $\left(f_{l}-f\right) /\left(f_{0}-f_{l}\right) \times 100 \%$, where $f_{0}$ is the fundamental oscillation frequency of a QCM quartz crystal, $f_{l}$ is the frequency after material deposition on that crystal and $f$ is the frequency of the film-coated crystal measured during the sensing experiment. The third overtone was used in the calculations of responses. Linear baseline corrections were applied in some sensing traces. The theoretical limits of detection (LOD) is estimated with the signal-to-noise ratio $(\mathrm{S} / \mathrm{N})>3 .{ }^{\mathrm{S} 2}$ The baseline noises were determined using the variation of the initial 100 points of baseline before NDMA exposure. Specifically, the initial 100 points were fitted with the polynomial equation $y=a x^{5}+b x^{4}+c x^{3}+d x^{2}+e x+f .^{\mathrm{S} 3}$ The $\mathrm{rms}_{\text {noise }}$ was then calculated according to Eq. S1 and Eq. S2. Finally, the LOD was calculated through Eq. S3. The reported LOD was an average of the values estimated from five different concentrations (100 ppb, $500 \mathrm{ppb}, 2,5$ and $10 \mathrm{ppm}$ ).

$V_{x^{2}}=\sum\left(y_{i}-y\right)^{2} \quad$ Eq. S1, where $y_{i}$ is the experimental value and $y$ is the corresponding value from the fit.

$r m s_{\text {noise }}=\sqrt{\frac{V_{x^{2}}}{N}} \quad$ Eq. S2, where $N$ is the number of baseline data points used for the fitting $(N=100)$.

LOD $(\mathrm{ppm})=3 \frac{r m s_{\text {noise }}}{\text { slope }}$ Eq. S3, where slope is obtained from the linear fitting of experimental concentration-dependent responses. 


\section{Synthesis}

Compounds $\mathbf{2}, \mathbf{3}$ and $\mathbf{5}$ were synthesized according to the known procedures. ${ }^{\mathrm{S} 4}$
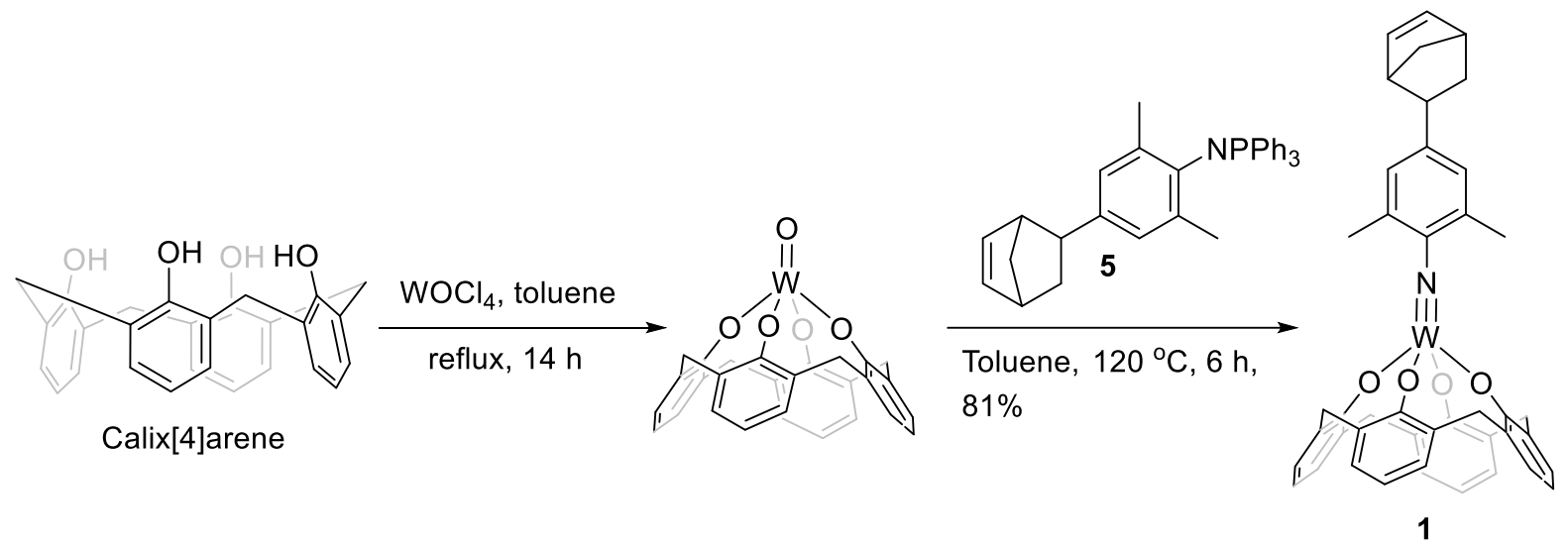

Compound 1 was synthesized using the similar method to that of compound 2. Calix[4]arene (354 mg, 0.835 $\mathrm{mmol}), \mathrm{WOCl}_{4}(300 \mathrm{mg}, 0.878 \mathrm{mmol})$ were charged into a flask. Dry toluene $(40 \mathrm{ml})$ was added under Ar. The mixture was refluxed at $125{ }^{\circ} \mathrm{C}$ overnight. After cooling, 5 (593 mg, $1.25 \mathrm{mmol}$ ) was added to the mixture. The mixture was further refluxed for $6 \mathrm{~h}$. The solvent was removed. The crude product was purified by column chromatography over silica gel (PE/DCM $2 / 1$ to $1 / 1$ ) to provide a yellow solid $(553 \mathrm{mg}, 81 \%) .{ }^{1} \mathrm{H}$ NMR (500 MHz, $\left.\mathrm{CD}_{2} \mathrm{Cl}_{2}\right) \delta 7.25-7.07(\mathrm{~m}, 10 \mathrm{H}), 6.68(\mathrm{t}, J=7.6 \mathrm{~Hz}, 4 \mathrm{H}), 6.28(\mathrm{dd}, J=5.7,3.1 \mathrm{~Hz}, 1 \mathrm{H})$, $6.18(\mathrm{dd}, J=5.6,2.9 \mathrm{~Hz}, 1 \mathrm{H}), 4.59(\mathrm{~d}, J=12.4 \mathrm{~Hz}, 4 \mathrm{H}), 3.30(\mathrm{~d}, J=12.4 \mathrm{~Hz}, 4 \mathrm{H}), 3.11(\mathrm{~s}, 6 \mathrm{H}), 3.00(\mathrm{~s}, 1 \mathrm{H})$, $2.94-2.83(\mathrm{~m}, 2 \mathrm{H}), 1.76-1.72(\mathrm{~m}, 1 \mathrm{H}), 1.69-1.56(\mathrm{~m}, 2 \mathrm{H}), 1.45-1.43(\mathrm{~m}, 1 \mathrm{H}) .{ }^{13} \mathrm{C}$ NMR $(126 \mathrm{MHz}$, $\left.\mathrm{CD}_{2} \mathrm{Cl}_{2}\right) \delta 157.81,157.65,147.28,140.34,140.32,137.80,137.64,131.50,128.63,126.39,122.87,49.20$, $46.18,43.79,42.79,34.34,32.42,18.47$. HR-MS (LC-QToF-ESI): calc for $[\mathrm{M}+\mathrm{H}]^{+}:$: 816.2310, Found: 816.2300 .

\section{General synthetic procedures for ROMP polymers.}

Compound $\mathbf{1}$ or $\mathbf{2}$ was dissolved in dichloromethane (DCM) in a glovebox. Grubbs catalyst (0.005 eq) was dissolved in DCM and was added to the monomer solution quickly. The mixture was stirred at room temperature for $3 \mathrm{~h}$ and quenched with ethyl vinyl ether. The solution was then dropped into methanol. The resulting solid was collected by filter and washed with methanol for three times. The obtained polymer was dried under vacuum at $80{ }^{\circ} \mathrm{C}$ overnight. PCalixH was obtained as a yellow solid (95 mg, 93\%). PCalixtBu was obtained as a yellow solid (93 $\mathrm{mg}, 87 \%$ ).

\section{Supplementary Figures and Tables}




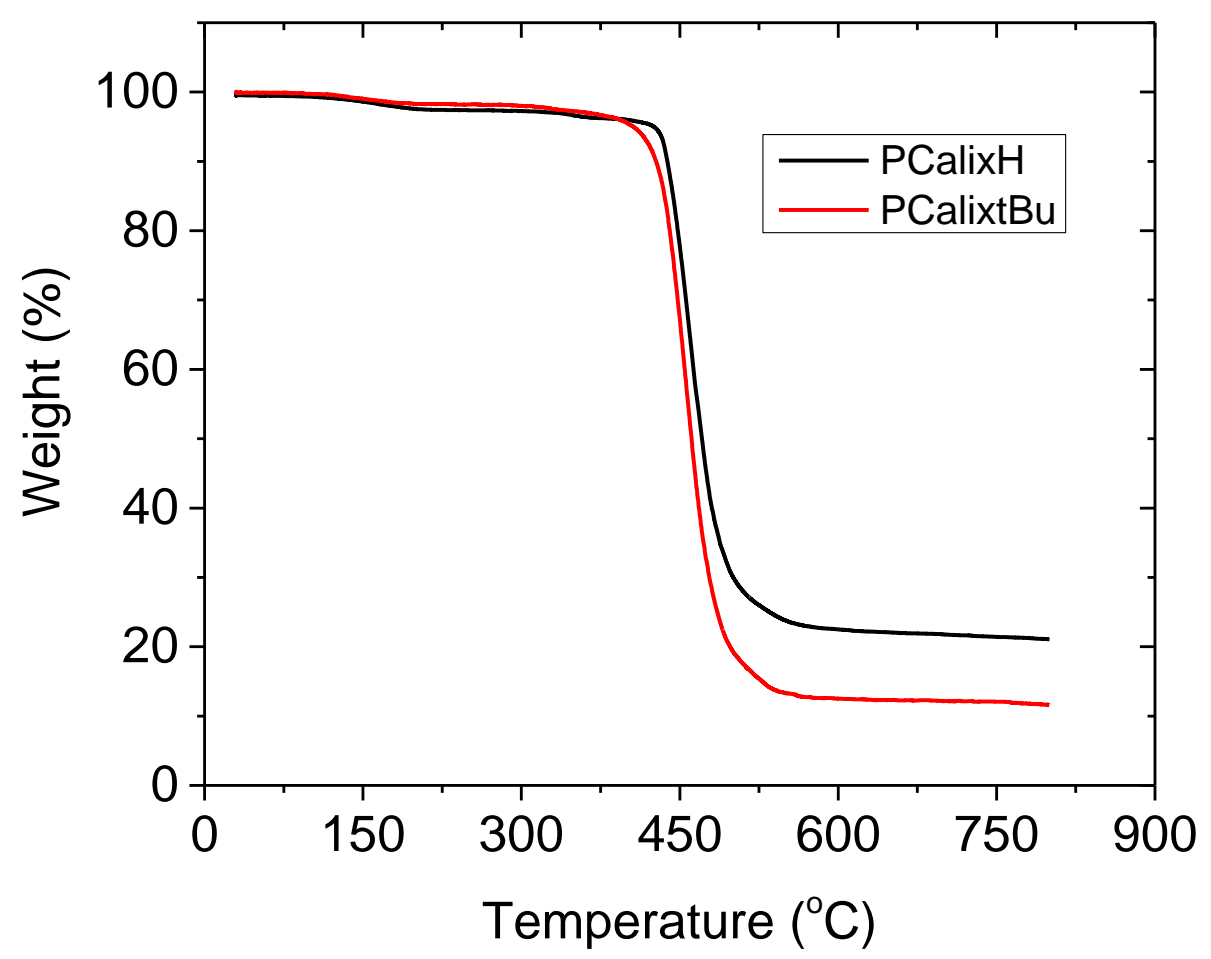

Figure S1. Thermal gravity analyses (TGA) of PCalixH and PCalixtBu.

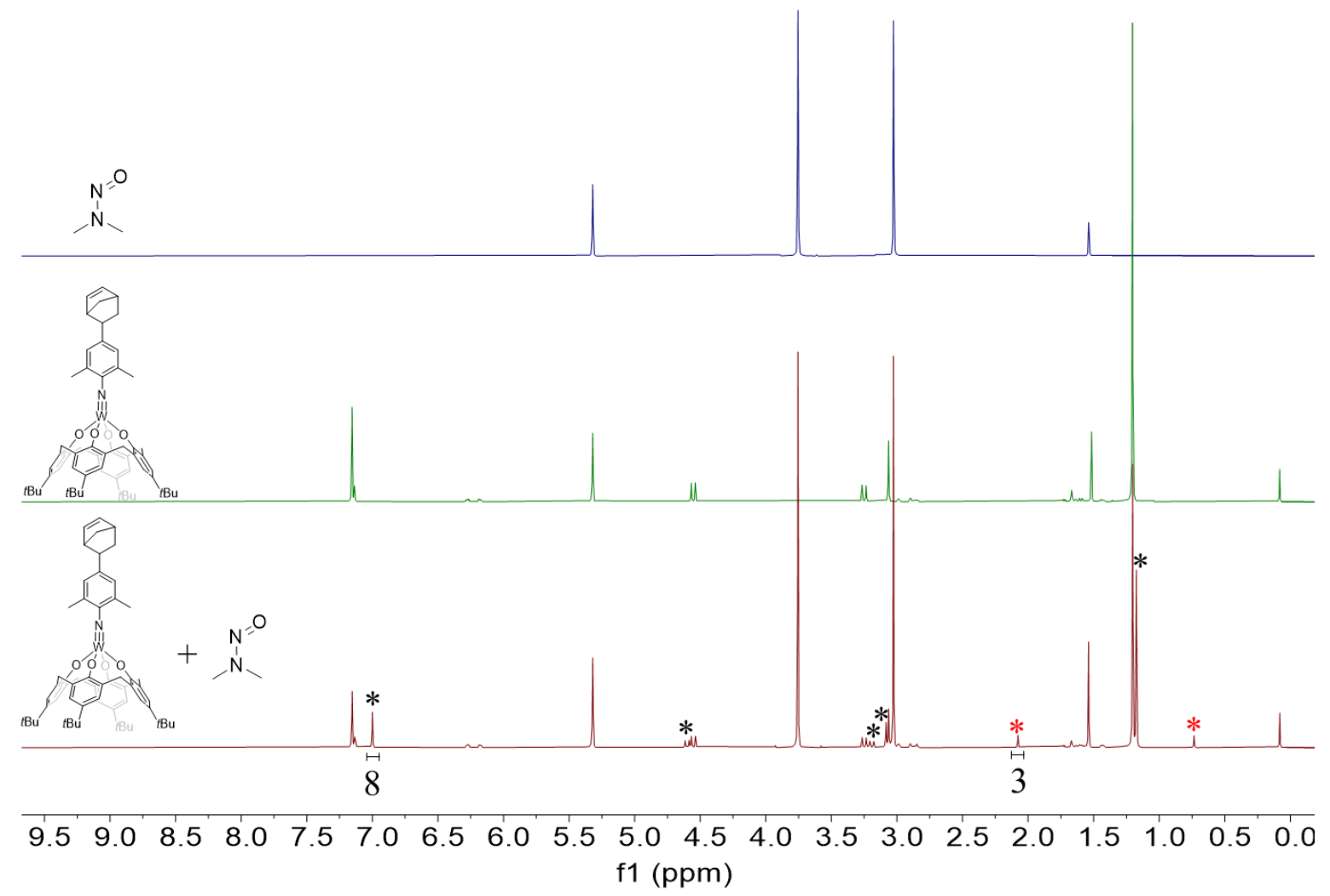

Figure S2. ${ }^{1} \mathrm{H}$ NMR of NDMA, 2 and their mixture in $\mathrm{CD}_{2} \mathrm{Cl}_{2}$. The signals of $\mathbf{2}$ and NDMA complex are marked with black stars for those from $\mathbf{2}$ and with red stars for those from NDMA. 
(a)

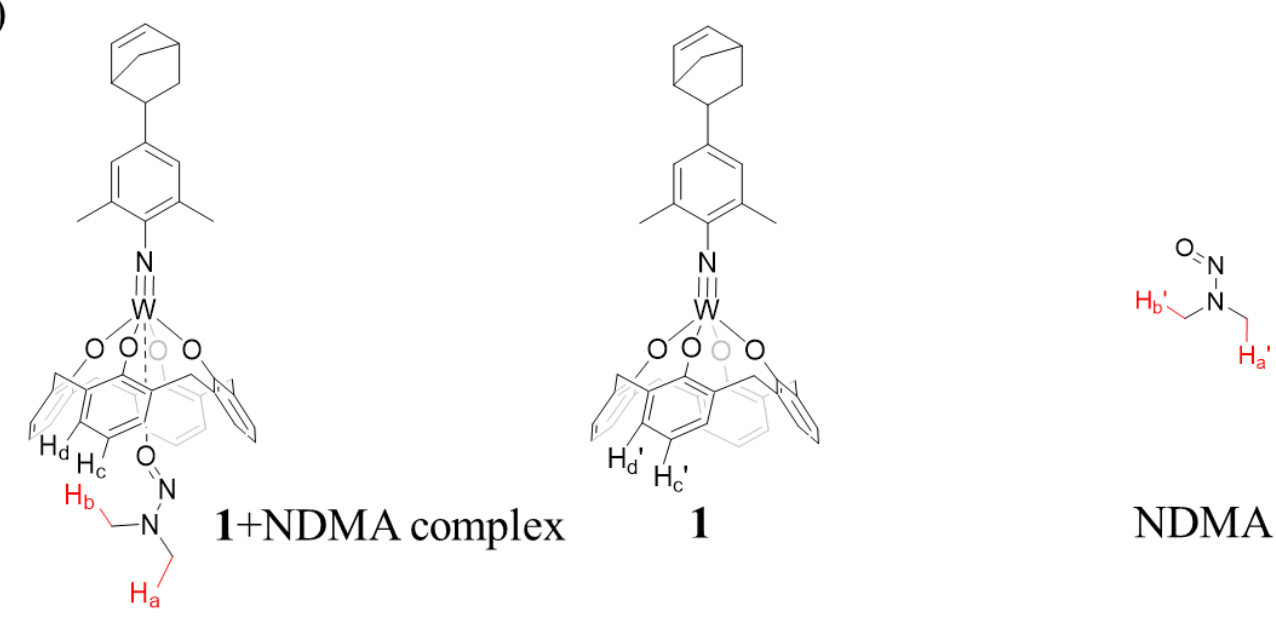

(b)
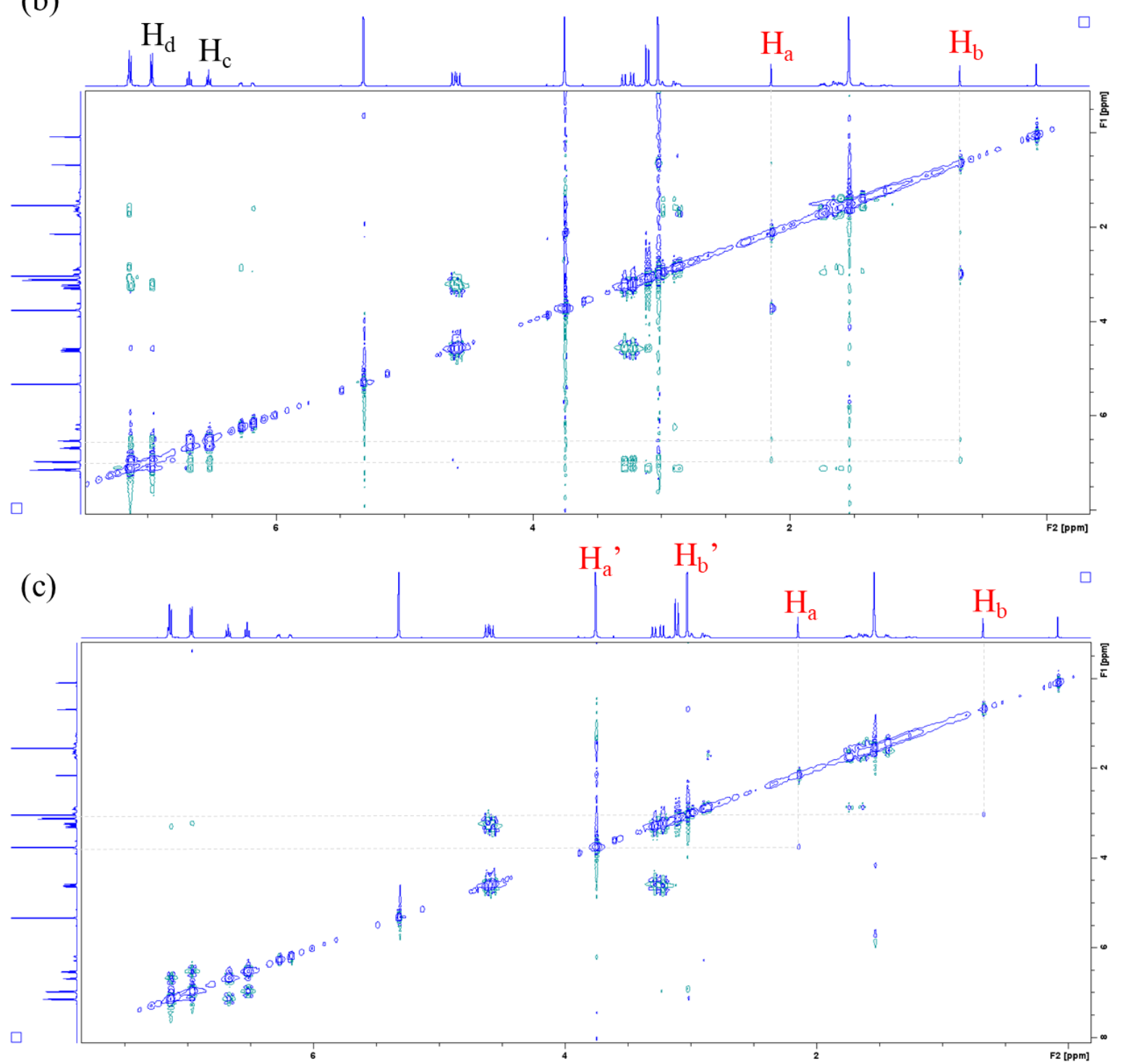

Figure S3. (a) Structures of $\mathbf{1}+$ NDMA complex, 1 and NDMA. (b) ${ }^{1} \mathrm{H}^{-1} \mathrm{H}$ nuclear overhauser effect spectroscopy (NOESY) (mixing time: $600 \mathrm{~ms}$ ) and (c) ${ }^{1} \mathrm{H}-{ }^{1} \mathrm{H}$ exchange spectroscopy (EXSY) (mixing time: $30 \mathrm{~ms}$ ) of mixture of 1 and NDMA in $\mathrm{CD}_{2} \mathrm{Cl}_{2}$. 

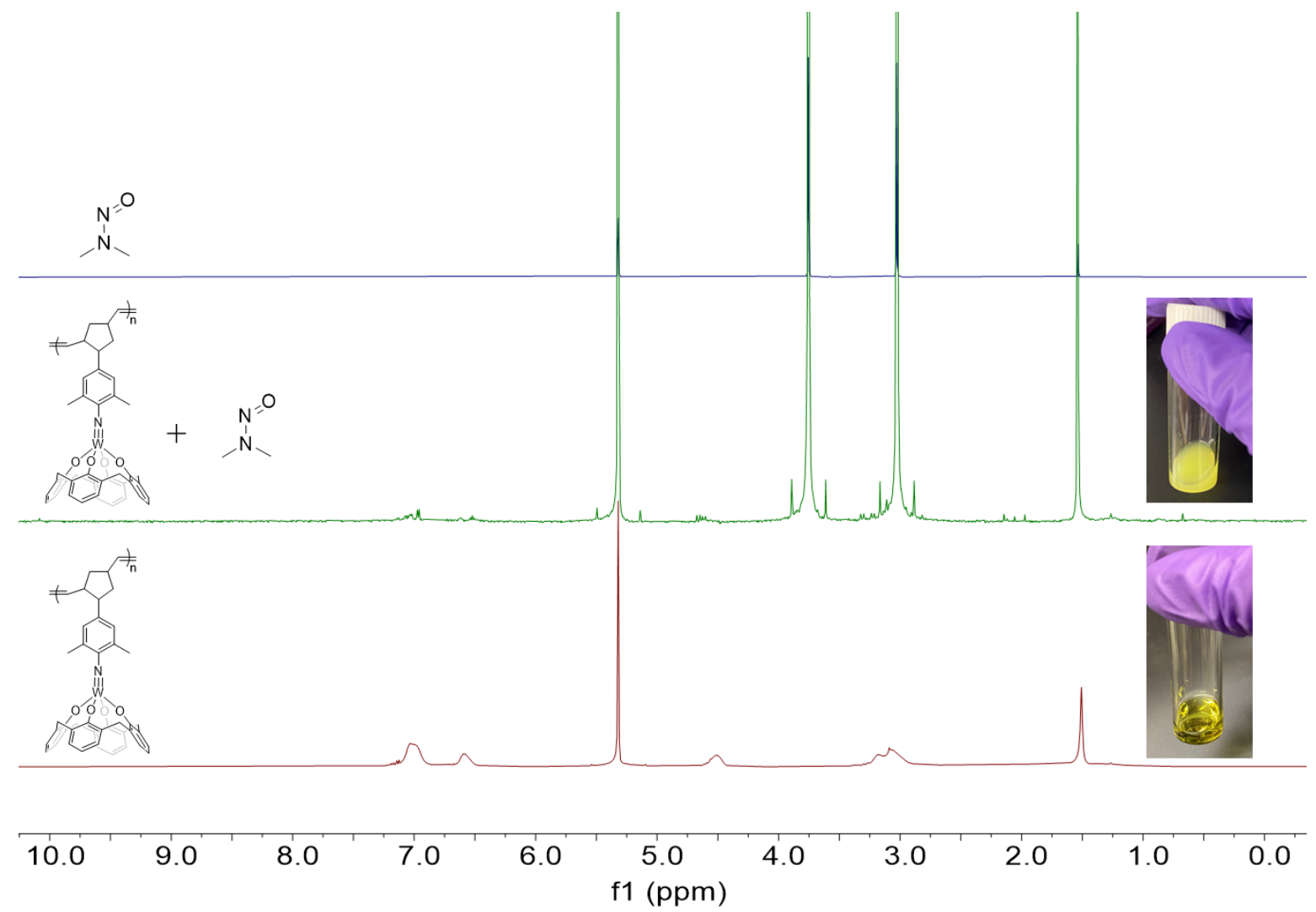

Figure S4. ${ }^{1} \mathrm{H}$ NMR of NDMA, PCalixtH and their mixture in $\mathrm{CD}_{2} \mathrm{Cl}_{2}$. The solubility of $\mathrm{PCalixH}$ in $\mathrm{CD}_{2} \mathrm{Cl}_{2}$ become low when NDMA was added.

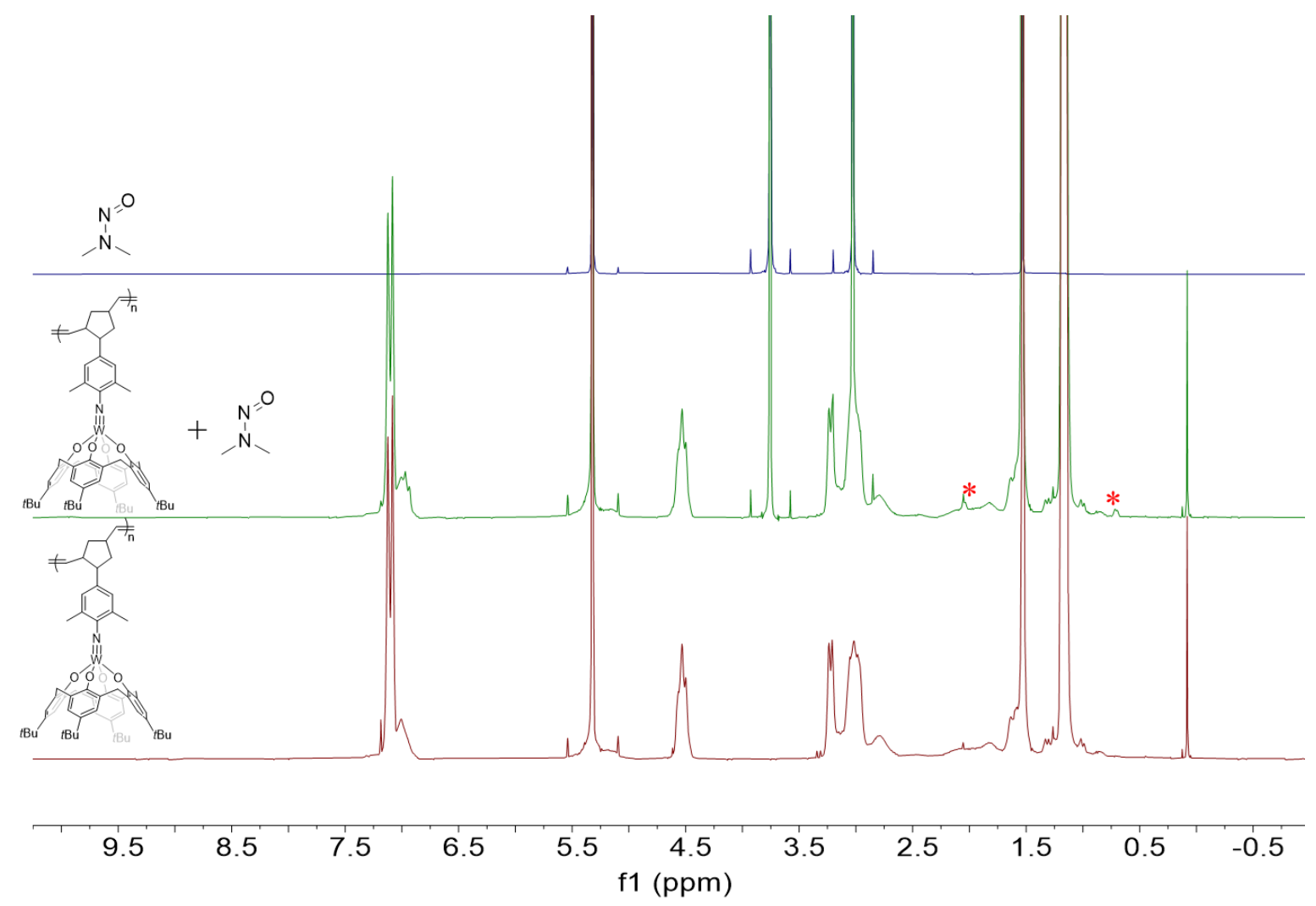

Figure S5. ${ }^{1} \mathrm{H}$ NMR of NDMA, PCalixtBu and their mixture in $\mathrm{CD}_{2} \mathrm{Cl}_{2}$. The signals of NDMA in the complex are marked with red stars. 
Table S1. Crystal data and structure refinement for $\mathbf{3}$ and NDMA complex.

\begin{tabular}{|c|c|}
\hline $\mathrm{CCDC}$ & 2103918 \\
\hline Empirical formula & $\mathrm{C}_{39} \mathrm{H}_{37} \mathrm{~N}_{3} \mathrm{O}_{5} \mathrm{~W}$ \\
\hline Formula weight & 811.56 \\
\hline Temperature/K & $100(2)$ \\
\hline Crystal system & orthorhombic \\
\hline Space group & Pbca \\
\hline $\mathrm{a} / \AA$ & $27.8186(7)$ \\
\hline $\mathrm{b} / \AA$ & $25.1486(7)$ \\
\hline $\mathrm{c} / \AA ̊$ & $27.8935(7)$ \\
\hline$\alpha /^{\circ}$ & 90 \\
\hline$\beta /{ }^{\circ}$ & 90 \\
\hline$\gamma /{ }^{\circ}$ & 90 \\
\hline Volume $/ \AA^{3}$ & 19514.3(9) \\
\hline $\mathrm{Z}$ & 24 \\
\hline$\rho_{\text {calc }} \mathrm{g} / \mathrm{cm}^{3}$ & 1.657 \\
\hline$\mu / \mathrm{mm}^{-1}$ & 3.603 \\
\hline $\mathrm{F}(000)$ & 9744.0 \\
\hline Crystal size $/ \mathrm{mm}^{3}$ & $0.365 \times 0.290 \times 0.180$ \\
\hline Radiation & $\operatorname{MoK} \alpha(\lambda=0.71073)$ \\
\hline $2 \Theta$ range for data collection $/{ }^{\circ}$ & 2.626 to 63.012 \\
\hline Index ranges & $\begin{array}{l}-40 \leq \mathrm{h} \leq 40,-36 \leq \mathrm{k} \leq 36,-41 \leq \\
1 \leq 41\end{array}$ \\
\hline Reflections collected & 910663 \\
\hline Independent reflections & $\begin{array}{l}32475\left[R_{\text {int }}=0.0610, R_{\text {sigma }}=\right. \\
0.0179]\end{array}$ \\
\hline Data/restraints/parameters & $32475 / 780 / 1432$ \\
\hline Goodness-of-fit on $\mathrm{F}^{2}$ & 1.072 \\
\hline Final R indexes $[\mathrm{I}>=2 \sigma(\mathrm{I})]$ & $\mathrm{R}_{1}=0.0220, \mathrm{wR}_{2}=0.0450$ \\
\hline Final $\mathrm{R}$ indexes [all data] & $\mathrm{R}_{1}=0.0324, \mathrm{wR}_{2}=0.0486$ \\
\hline Largest diff. peak/hole / e $\AA^{-3}$ & $0.66 /-1.59$ \\
\hline
\end{tabular}



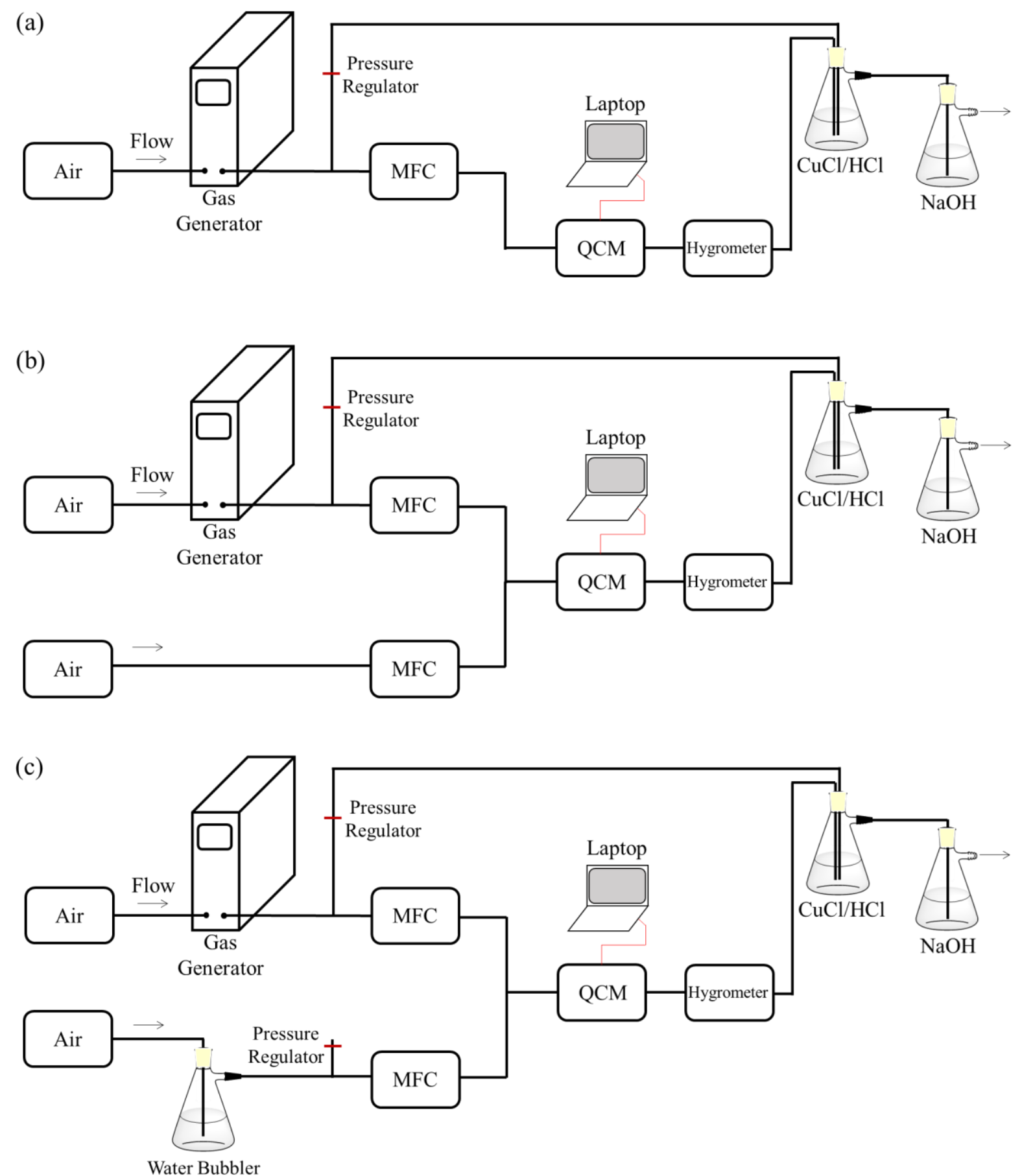

Figure S6. Sensing setups for (a) ppm and (b) ppb level gas analytes and (c) for controlling humidity. The sensing measurements should be performed in a fume hood. The sensing exhaust gas was quenched by cuprous chloride in $6 \mathrm{M}$ hydrochloric acid..$^{\mathrm{S} 5}$ The exhaust acid gas was further quenched by sodium hydroxide water solution. 
(a)

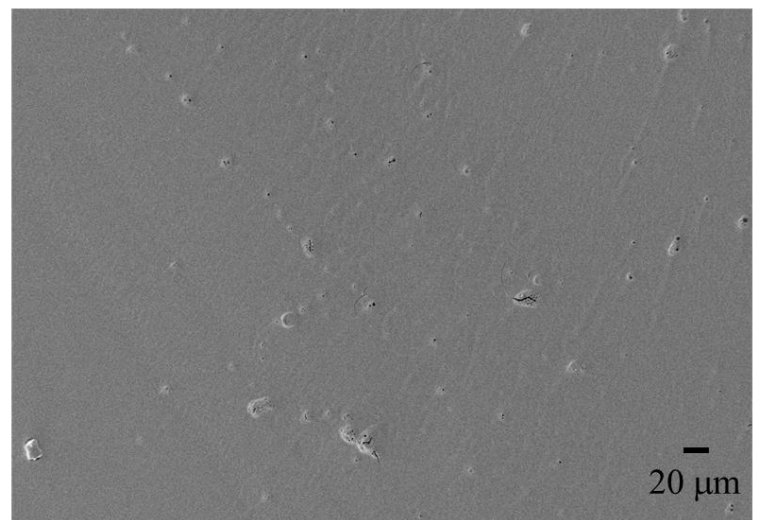

(b)

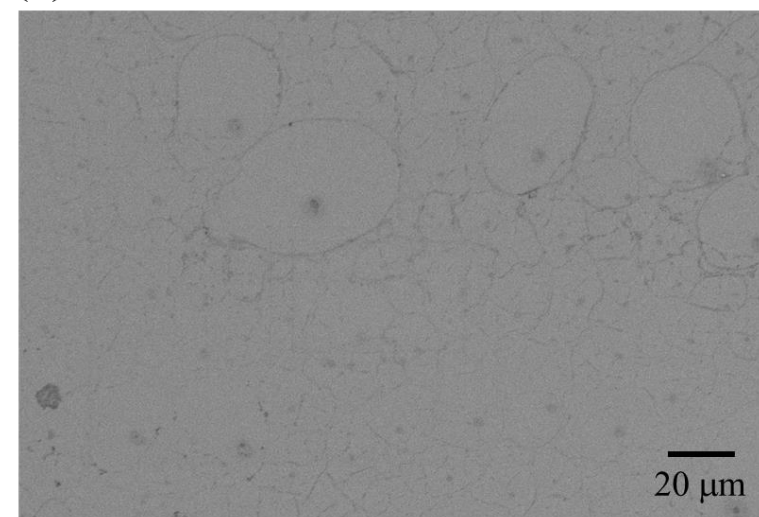

Figure S7. SEM images of dropcast films of (a) PCalixH and (b) PCalixtBu.

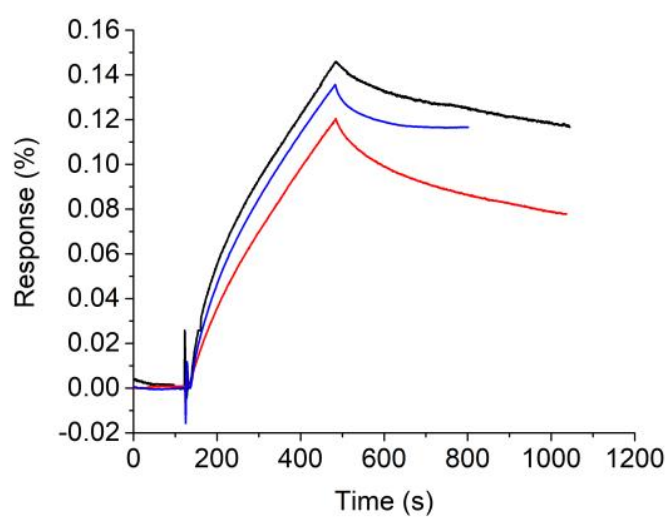

Figure S8. Sensing traces of PCalixH to 5 ppm NDMA.

(a)
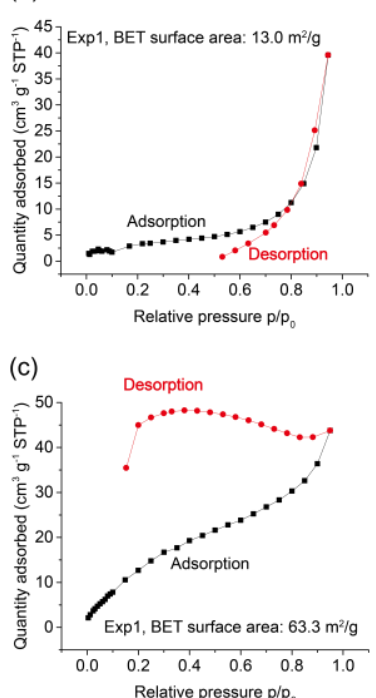
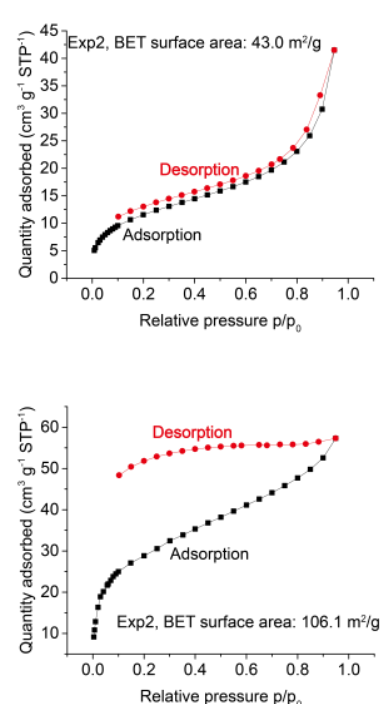
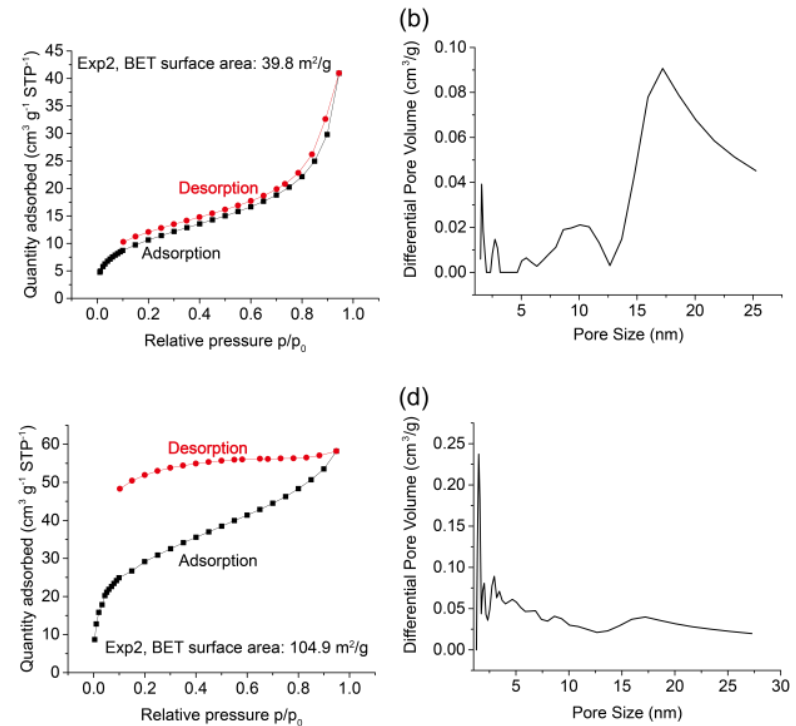

Figure S9. $\mathrm{N}_{2}$ adsorption-desorption isotherm of (a) PCalixH and (c) PCalixtBu. Pore size distributions of (b) PCalixH and (d) PCalixtBu. 
(a)

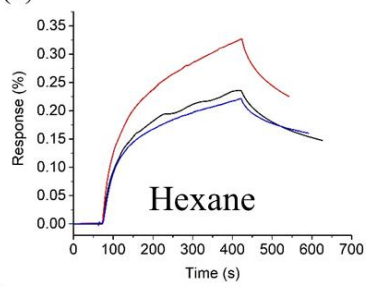

(e)

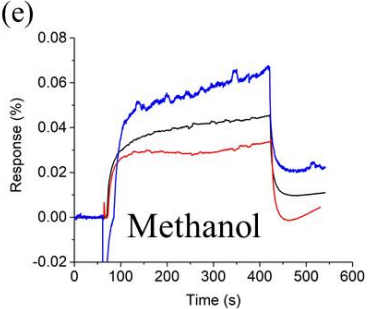

(i)

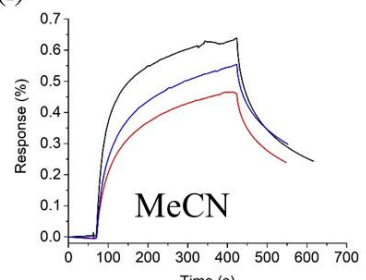

(b)

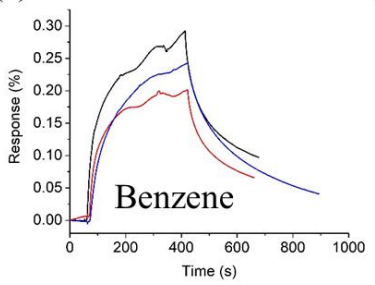

(f)

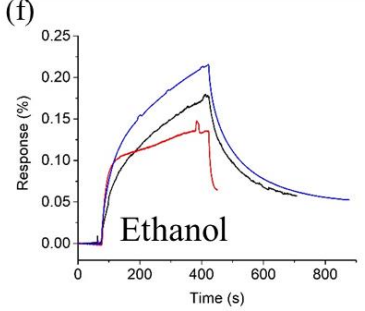

(j)

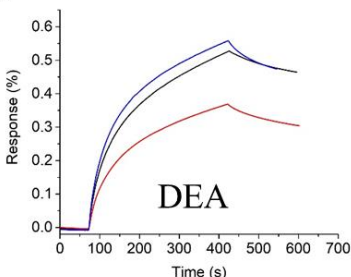

(c)

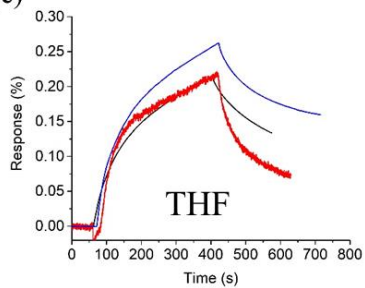

(g)

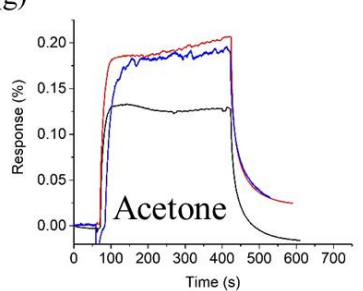

(h)
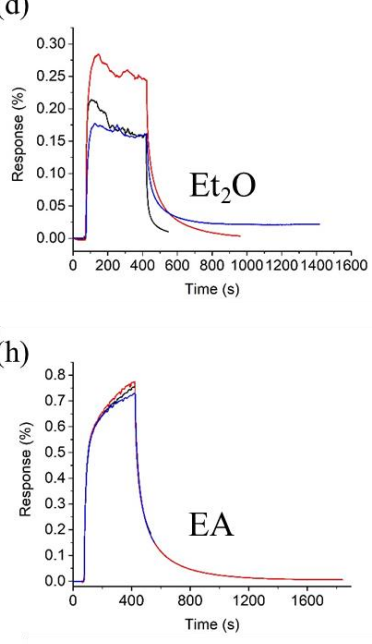

(k)

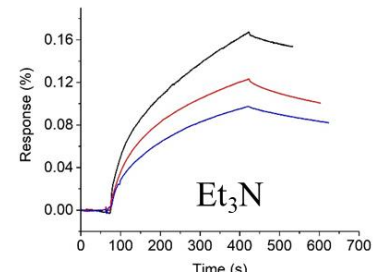

Figure S10. Sensing traces for (a) hexane (100 ppm), (b) benzene (50 ppm), (c) THF (60 ppm), (d) $\mathrm{Et}_{2} \mathrm{O}$ (150 ppm), (e) methanol (100 ppm), (f) ethanol (100 ppm), (g) acetone (130 ppm), (h) EA (100 ppm), (i) $\mathrm{MeCN}(50 \mathrm{ppm}),(\mathrm{j}) \mathrm{DEA}(50 \mathrm{ppm})$, and $(\mathrm{k}) \mathrm{Et}_{3} \mathrm{~N}$ (100 ppm). All sensing was measured for three times. The exposure time is $6 \mathrm{~min}$.
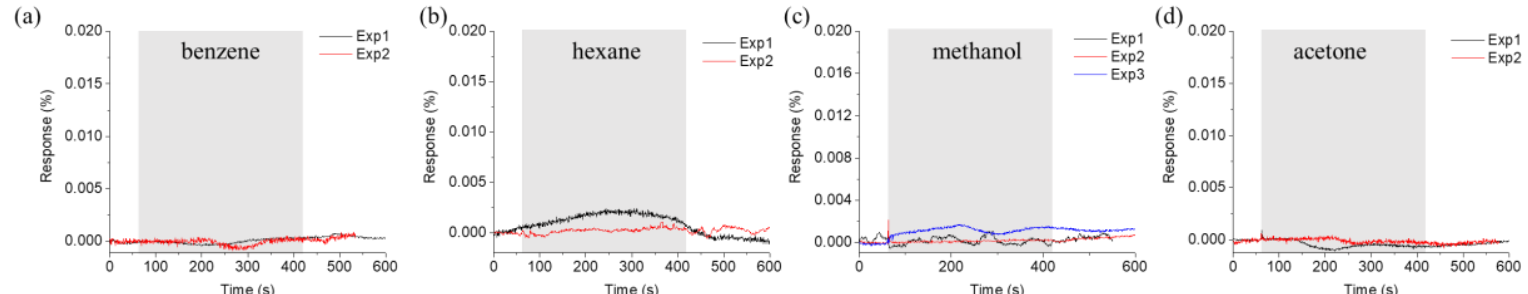

Figure S11. Sensing traces of 2-3 independent measurements for (a) benzene (100 ppb), (b) hexane (100 $\mathrm{ppb}),(\mathrm{c})$ methanol (100 ppb), (d) acetone (100 ppb). The exposure time is $6 \mathrm{~min}$. 
(a)

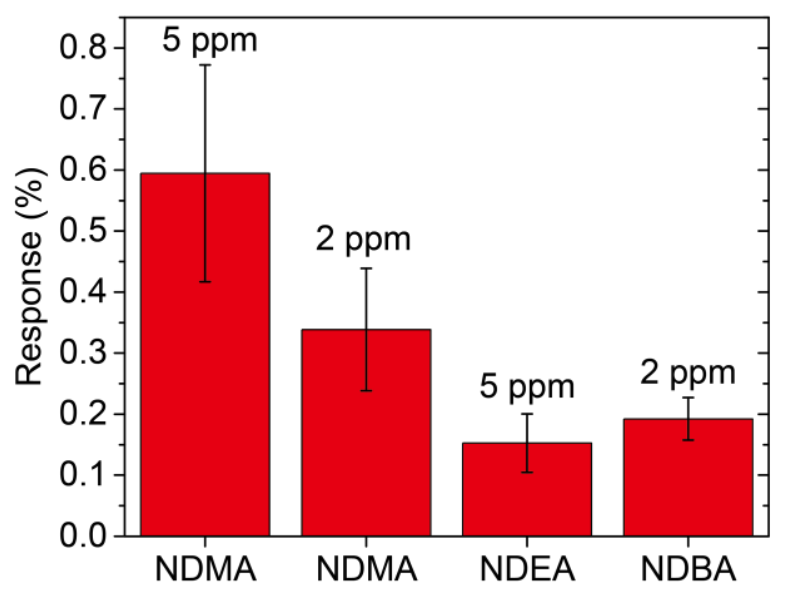

(b)

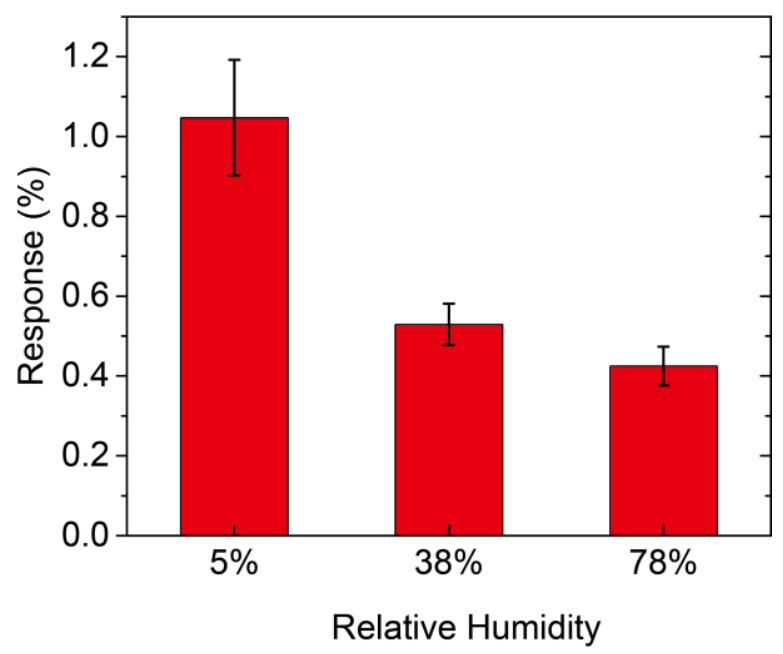

Figure S12. (a) Sensing response of PCalixtBu to NDEA and NDBA. (b) The responses of PCalixtBu to 10 ppm NDMA with different relative humidity.

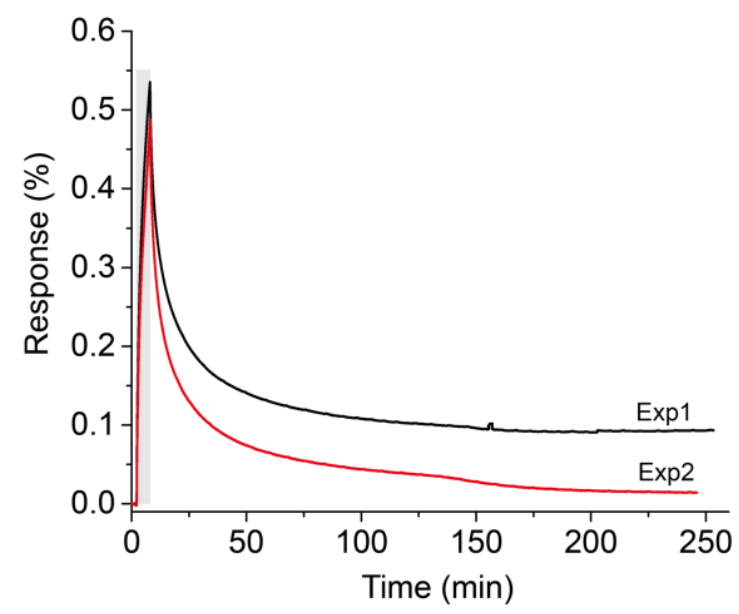

Figure S13. Sensor reversibility. The area highlighted by light grey indicates exposure to 5 ppm NDMA.

\section{Theoretical calculations}

Geometric optimizations in the gas phase were carried out using the $\omega \mathrm{B} 97 \mathrm{X}-\mathrm{D}^{\mathrm{S6}}$ functional with the 6-31G(d) basic set for C, H, O and N and the LanL2DZ basic set for $\mathrm{W}$ on Gaussian 09, Revision D.01 ${ }^{\mathrm{S7}}$ Harmonic vibrational frequency calculations were performed for all of the stationary points to confirm them as local minima. 
(a)

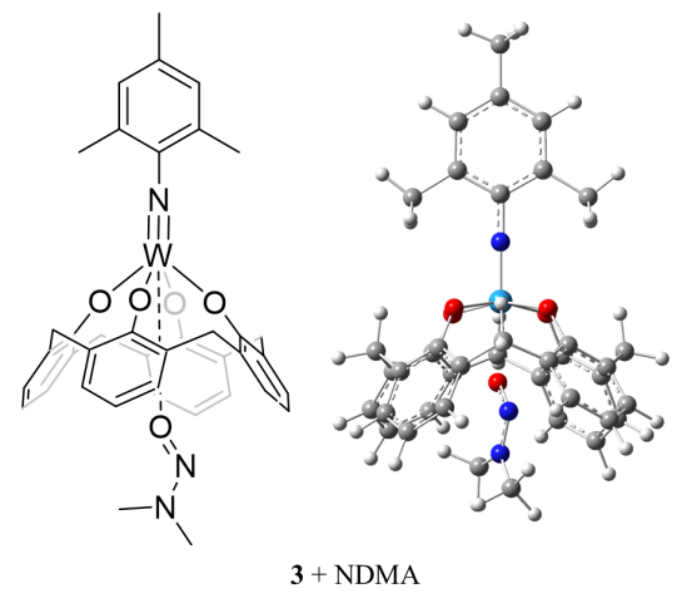

(b)

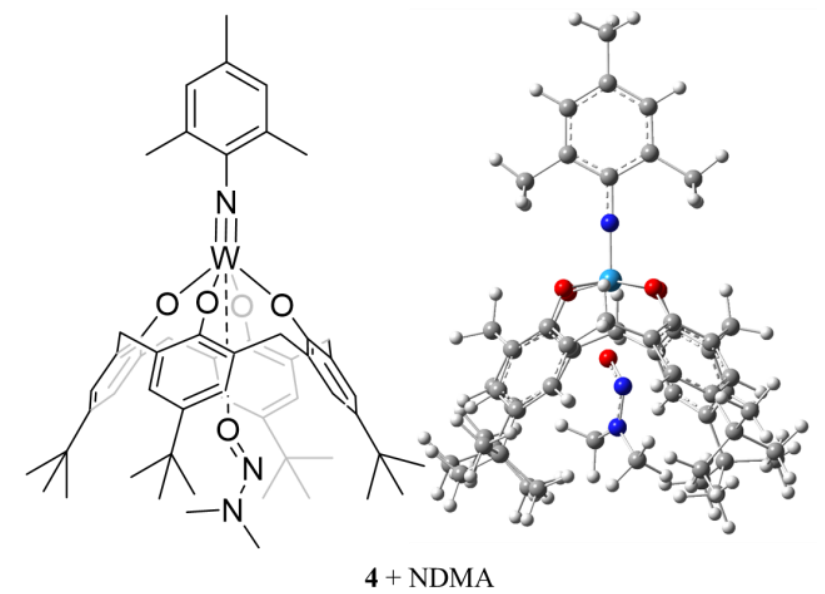

Figure S14. Molecular structures and modeling of (a) 3 + NDMA and (b) 4 + NDMA complexes.

\section{NDMA}

$E=-264.387196$ Hartree

$\begin{array}{lrrr}\mathrm{N} & -0.33601500 & -0.00312200 & -0.00099600 \\ \mathrm{C} & -0.11888700 & 1.42992300 & 0.00008100 \\ \mathrm{C} & -1.65661900 & -0.58925500 & 0.00036400 \\ \mathrm{~N} & 0.69578400 & -0.83866900 & -0.00027800 \\ \mathrm{O} & 1.78586600 & -0.28493200 & 0.00039800 \\ \mathrm{H} & 0.45465000 & 1.72675000 & -0.88317500 \\ \mathrm{H} & 0.45148000 & 1.72613800 & 0.88563200 \\ \mathrm{H} & -1.09011900 & 1.92638800 & -0.00151000 \\ \mathrm{H} & -2.21686000 & -0.28890300 & 0.89269900 \\ \mathrm{H} & -1.53288900 & -1.67331500 & 0.00034800 \\ \mathrm{H} & -2.21853900 & -0.28906700 & -0.89093100\end{array}$

$\mathrm{H}_{2} \mathrm{O}$

$\mathrm{E}=-76.387063$ Hartree

$\begin{array}{lrrr}\mathrm{O} & 0.00000000 & 0.00000000 & 0.11835300 \\ \mathrm{H} & 0.00000000 & -0.75982300 & -0.47341400 \\ \mathrm{H} & 0.00000000 & 0.75982300 & -0.47341400\end{array}$

Compound 3

$\mathrm{E}=-1851.722125$ Hartree

$\begin{array}{lrrr}\mathrm{W} & -0.51249300 & -0.00412000 & -0.00184000 \\ \mathrm{O} & -0.10840400 & 1.31080200 & -1.31777500 \\ \mathrm{C} & 1.03321500 & 1.83840800 & -1.84174500 \\ \mathrm{C} & 1.58213200 & 2.98917800 & -1.25970800 \\ \mathrm{C} & 2.72066800 & 3.54360700 & -1.84195000 \\ \mathrm{H} & 3.15580000 & 4.43637100 & -1.39949400 \\ \mathrm{C} & 3.29796200 & 2.97699600 & -2.97254100\end{array}$




$$
4.18174900
$$

2.73261300

3.17674500

1.59416200

0.99988900

1.18960200

$-0.08391900$

$-0.10087100$

1.04438500

1.60212900

2.74467800

3.18652400

3.31696500

4.20379700

2.74266500

3.18317400

1.60035400

0.99860400

1.18632000

$-0.08517200$

$-0.10328900$

1.04057200

1.59760800

2.73843700

3.17978200

3.31028600

4.19601300

2.73695300

3.17681500

1.59577500

0.99212600

1.17949400

$-0.09138200$

$-0.11040800$

1.02922200

1.58727700

2.72362000

3.16561300

3.28958400

4.17166100

2.71515200

3.15077700

1.57887800

0.97686000
3.42413300

1.83824200

1.39283100

1.25193300

$-0.00670200$

$-0.00860300$

8860300

$-0.00990300$

$-1.32192300$

$-1.84422900$

$-1.25880700$

$-1.83999400$

$-1.39526200$

$-2.97286800$

$-3.41616300$

$-3.53866800$

$-4.42692200$

$-2.98909000$

$-3.58691700$

$-4.66582300$

$-3.43909500$

$-1.31603800$

$-1.83614800$

$-2.98368500$

$-3.53102900$

$-4.42127600$

$-2.96047200$

$-3.40204500$

$-1.82504200$

$-1.37665000$

$-1.24591700$

0.00869100

0.01132200

0.00465000

1.31608400

1.84660800

1.26517000

1.85470700

1.41322500

2.99172700

3.44143600

3.55328000

4.44467300

2.99541100

3.58900900
$-3.41674500$

$-3.53483700$

$-4.42166700$

$-2.98446300$

$-3.58193700$

$-4.66047000$

$-3.43666200$

$-1.31249100$

$-1.83395200$

$-2.97876400$

$-3.52609600$

$-4.41440500$

$-2.95897400$

$-3.40095800$

$-1.82644800$

$-1.38026400$

$-1.24702900$

0.00781300

0.01041200

0.00628300

1.31543800

1.84205300

1.26136600

1.84572600

1.40436800

2.97710200

3.42300700

3.53804800

4.42550500

2.98562600

3.58225900

4.66119900

3.43472000

1.30921300

1.83443600

2.98112500

3.53239900

4.42225800

2.96722300

3.41222800

1.83264100

1.38790900

1.24927500

$-0.00747900$ 


\begin{tabular}{lrrr}
$\mathrm{H}$ & -0.10607200 & 3.43494000 & -0.00854100 \\
$\mathrm{H}$ & 1.15824100 & 4.66899800 & -0.00999500 \\
$\mathrm{~N}$ & -2.21232100 & -0.00905800 & -0.00366100 \\
$\mathrm{C}$ & -3.59470300 & -0.00927200 & -0.00281100 \\
$\mathrm{C}$ & -4.27258400 & -1.23697000 & 0.00070100 \\
$\mathrm{C}$ & -5.66506900 & -1.20818300 & 0.00410200 \\
$\mathrm{C}$ & -6.37579600 & -0.00809500 & 0.00309700 \\
$\mathrm{C}$ & -5.66228800 & 1.19373000 & 0.00106000 \\
$\mathrm{C}$ & -4.27263900 & 1.22137100 & -0.00262400 \\
$\mathrm{C}$ & -3.49106500 & 2.50760700 & -0.00385900 \\
$\mathrm{C}$ & -3.49384000 & -2.52489700 & 0.00322400 \\
$\mathrm{C}$ & -7.88364600 & 0.00417300 & -0.00968500 \\
$\mathrm{H}$ & -6.20822800 & -2.15066700 & 0.00851100 \\
$\mathrm{H}$ & -6.20606300 & 2.13624600 & 0.00317200 \\
$\mathrm{H}$ & -2.84181400 & 2.57109600 & -0.88456800 \\
$\mathrm{H}$ & -2.83949000 & 2.57165500 & 0.87508200 \\
$\mathrm{H}$ & -4.15996800 & 3.37260000 & -0.00332300 \\
$\mathrm{H}$ & -2.84354100 & -2.59173500 & -0.87643300 \\
$\mathrm{H}$ & -4.16450300 & -3.38852400 & 0.00468200 \\
$\mathrm{H}$ & -2.84375000 & -2.58858300 & 0.88329500 \\
$\mathrm{H}$ & -8.29481100 & -0.99285300 & 0.17347100 \\
$\mathrm{H}$ & -8.26556300 & 0.34956600 & -0.97786900 \\
$\mathrm{H}$ & -8.28019400 & 0.68006300 & 0.75578000 \\
& & & \\
\hline
\end{tabular}

Compound 3 + NDMA

$E=-2116.161717$ Hartree

$\begin{array}{lrrr}\mathrm{W} & -0.79240700 & -0.00998000 & -0.00003000 \\ \mathrm{O} & -0.54977100 & -0.40107900 & 1.87656000 \\ \mathrm{C} & 0.60272400 & -0.51114700 & 2.57335000 \\ \mathrm{C} & 1.23425100 & -1.76300700 & 2.65163500 \\ \mathrm{C} & 2.42939400 & -1.85245500 & 3.36345900 \\ \mathrm{H} & 2.93010800 & -2.81564000 & 3.43310400 \\ \mathrm{C} & 2.97885400 & -0.73875600 & 3.99182500 \\ \mathrm{H} & 3.90360900 & -0.82979300 & 4.55382500 \\ \mathrm{C} & 2.32744000 & 0.48928300 & 3.90854200 \\ \mathrm{H} & 2.75257700 & 1.36247300 & 4.39921200 \\ \mathrm{C} & 1.13872300 & 0.62937400 & 3.19388200 \\ \mathrm{C} & 0.47391600 & 1.98955300 & 3.04299000 \\ \mathrm{H} & 0.61672300 & 2.55025900 & 3.97346800 \\ \mathrm{H} & -0.60125900 & 1.85206400 & 2.90090800 \\ \mathrm{O} & -0.53490100 & 1.85294500 & 0.39326200 \\ \mathrm{C} & 0.53832800 & 2.64327100 & 0.59044700 \\ \mathrm{C} & 1.03693100 & 2.80747900 & 1.89258600 \\ \mathrm{C} & 2.08545600 & 3.70615200 & 2.08482100\end{array}$


$\mathrm{H}$

C

$\mathrm{H}$

C

$\mathrm{H}$

C

C

$\mathrm{H}$

$\mathrm{H}$

$\mathrm{O}$

$\mathrm{C}$

C

C

$\mathrm{H}$

C

$\mathrm{H}$

C

$\mathrm{H}$

C

C

$\mathrm{H}$

$\mathrm{H}$

$\mathrm{O}$

C

C

C

$\mathrm{H}$

C

$\mathrm{H}$

C

$\mathrm{H}$

C

C

$\mathrm{H}$

$\mathrm{H}$

$\mathrm{O}$

$\mathrm{N}$

$\mathrm{N}$

C

$\mathrm{H}$

$\mathrm{H}$

$\mathrm{H}$

C

$\mathrm{H}$
2.47142400

2.63864300

3.44125500

2.15750500

2.59783300

1.11429100

0.63520900

0.84282800

$-0.44795600$

$-0.49907400$

0.67976100

1.29822800

2.54212500

3.03571100

3.14946400

4.11110800

2.50582400

2.97475500

1.26780000

0.60761100

0.79629000

$-0.47340800$

$-0.48990300$

0.60455000

1.14118400

2.24880500

2.66857800

2.83282100

3.69088000

2.30614800

2.76475300

1.18543000

0.63576700

$-0.44966000$

0.83870300

1.51393400

3.59111900

2.40193800

4.64918400

5.36813300

5.14729500

4.19081300

3.86770400

3.42163100
3.85846000

3.09043000

$4.40880100 \quad 1.01740200$

$5.11989100 \quad 1.18839100$

$4.19177600 \quad-0.27148600$

$4.72407200-1.11175600$

$3.29719000 \quad-0.51003000$

$2.99854900 \quad-1.91928500$

$3.87128100 \quad-2.54874400$

$2.84786600-1.91154000$

$0.38872400-1.87319900$

$\begin{array}{ll}0.52268900 & -2.51869200\end{array}$

$1.78417400 \quad-2.55565200$

$1.88783100-3.17540500$

$2.85679900 \quad-3.20688300$

$0.77971800 \quad-3.76155800$

$0.88293400 \quad-4.25608100$

$-0.45380800 \quad-3.73185100$

$-1.32097200 \quad-4.19207700$

$-0.60844100 \quad-3.10890000$

$-1.97766200 \quad-3.00674600$

$-2.52426800 \quad-3.93756600$

$-1.85069000 \quad-2.90856000$

$-1.86485800 \quad-0.39359500$

$-2.63760800 \quad-0.55522600$

$-2.79454100-1.84285500$

$-3.62448500 \quad-1.99432000$

$-3.76926300 \quad-2.98772800$

$-4.25363700 \quad-0.89675800$

$-4.90547300 \quad-1.03298400$

$-4.04755000 \quad 0.37456000$

$-4.52924500 \quad 1.23534400$

$-3.24084700 \quad 0.56986800$

$-2.98099300 \quad 1.96255500$

$-2.86039200 \quad 1.90622500$

$-3.86109800 \quad 2.58310200$

$0.18067300 \quad 0.15245000$

$-0.10719800 \quad-0.17636600$

$-0.52364300 \quad-0.38474600$

$-0.87660000 \quad-0.80608000$

$-1.19501900 \quad-0.04655500$

$-0.25960800 \quad-1.55938700$

$-1.74725100 \quad-1.27558400$

$1.09108800 \quad 0.60279500$

$0.98876600 \quad 1.59427900$ 


$\begin{array}{crrc}\mathrm{H} & 3.42865800 & 1.95889200 & 0.10587200 \\ \mathrm{H} & 4.94940300 & 1.20094400 & 0.67813600 \\ \mathrm{~N} & -2.50145200 & -0.02478200 & -0.02910700 \\ \mathrm{C} & -3.87842600 & -0.03230700 & -0.05189800 \\ \mathrm{C} & -4.55323700 & 1.00568100 & -0.71269600 \\ \mathrm{C} & -5.94547500 & 0.97369000 & -0.72239600 \\ \mathrm{C} & -6.66115200 & -0.04813100 & -0.09855100 \\ \mathrm{C} & -5.95351100 & -1.06485200 & 0.54937000 \\ \mathrm{C} & -4.56437800 & -1.08029000 & 0.58723800 \\ \mathrm{C} & -3.78816600 & -2.16774500 & 1.28075200 \\ \mathrm{C} & -3.76861500 & 2.10178400 & -1.38275200 \\ \mathrm{C} & -8.16916600 & -0.06028400 & -0.10975700 \\ \mathrm{H} & -6.48468200 & 1.76997200 & -1.23119700 \\ \mathrm{H} & -6.50174200 & -1.86847700 & 1.03731000 \\ \mathrm{H} & -3.15879400 & -1.75277900 & 2.07599600 \\ \mathrm{H} & -3.11501500 & -2.67541300 & 0.58102900 \\ \mathrm{H} & -4.46068800 & -2.91037500 & 1.71930700 \\ \mathrm{H} & -3.12955300 & 2.62282600 & -0.66115400 \\ \mathrm{H} & -4.43544500 & 2.83216700 & -1.84964700 \\ \mathrm{H} & -3.10363600 & 1.69325900 & -2.15198600 \\ \mathrm{H} & -8.57002600 & 0.72964000 & -0.75180400 \\ \mathrm{H} & -8.57292600 & 0.09103500 & 0.89835300 \\ \mathrm{H} & -8.55534100 & -1.01883800 & -0.47425100\end{array}$

Compound $\mathbf{3}+\mathrm{H}_{2} \mathrm{O}$

$E=-1928.158003$ Hartree

$\begin{array}{lrrr}\mathrm{W} & 0.50640400 & -0.00090700 & 0.00553200 \\ \mathrm{O} & 0.19974600 & 1.67525800 & 0.87514400 \\ \mathrm{C} & -0.92664900 & 2.34620400 & 1.21795400 \\ \mathrm{C} & -1.47971000 & 3.25881400 & 0.30903600 \\ \mathrm{C} & -2.61390000 & 3.96928000 & 0.69714400 \\ \mathrm{H} & -3.04922000 & 4.68590400 & 0.00429700 \\ \mathrm{C} & -3.18979100 & 3.77672400 & 1.94836300 \\ \mathrm{H} & -4.06959300 & 4.34310900 & 2.23801900 \\ \mathrm{C} & -2.62962800 & 2.85609400 & 2.82776700 \\ \mathrm{H} & -3.07844400 & 2.69967000 & 3.80607000 \\ \mathrm{C} & -1.49475500 & 2.12548900 & 2.48015800 \\ \mathrm{C} & -0.91349900 & 1.08463200 & 3.42040100 \\ \mathrm{H} & 0.16989900 & 1.02887800 & 3.28562000 \\ \mathrm{H} & -1.10319100 & 1.39874000 & 4.45255900 \\ \mathrm{O} & 0.20747900 & -0.89092800 & 1.69226800 \\ \mathrm{C} & -0.93821600 & -1.21555500 & 2.33161200 \\ \mathrm{C} & -1.52096300 & -0.29192100 & 3.21741400 \\ \mathrm{C} & -2.69503200 & -0.65932700 & 3.87099300\end{array}$




\begin{tabular}{|c|c|c|c|}
\hline $\mathrm{H}$ & -3.15330600 & 0.04482600 & 4.56122000 \\
\hline $\mathrm{C}$ & -3.28795800 & -1.89914300 & 3.65125200 \\
\hline $\mathrm{H}$ & -4.20181800 & -2.16705500 & 4.17224300 \\
\hline $\mathrm{C}$ & -2.70392900 & -2.79205800 & 2.76025200 \\
\hline $\mathrm{H}$ & -3.16450000 & -3.76084200 & 2.58219400 \\
\hline $\mathrm{C}$ & -1.52460600 & -2.46980100 & 2.08540100 \\
\hline $\mathrm{C}$ & -0.91025700 & -3.42827300 & 1.07855500 \\
\hline $\mathrm{H}$ & -1.10137300 & -4.45438700 & 1.41056400 \\
\hline $\mathrm{H}$ & 0.17286500 & -3.28429300 & 1.04905700 \\
\hline $\mathrm{O}$ & 0.22136200 & -1.69590800 & -0.87884100 \\
\hline $\mathrm{C}$ & -0.90777100 & -2.35185800 & -1.22487700 \\
\hline $\mathrm{C}$ & -1.48807900 & -3.25128000 & -0.31424800 \\
\hline $\mathrm{C}$ & -2.63976700 & -3.93548600 & -0.70159000 \\
\hline $\mathrm{H}$ & -3.09170600 & -4.63917100 & -0.00640000 \\
\hline $\mathrm{C}$ & -3.21278700 & -3.73208200 & -1.95226900 \\
\hline $\mathrm{H}$ & -4.10618800 & -4.27748400 & -2.23985700 \\
\hline $\mathrm{C}$ & -2.63278000 & -2.82421600 & -2.83197600 \\
\hline $\mathrm{H}$ & -3.08025700 & -2.65581500 & -3.80872100 \\
\hline $\mathrm{C}$ & -1.48021300 & -2.11872700 & -2.48697400 \\
\hline $\mathrm{C}$ & -0.88989000 & -1.08111900 & -3.42544800 \\
\hline $\mathrm{H}$ & 0.19230700 & -1.02737500 & -3.28308600 \\
\hline $\mathrm{H}$ & -1.07550000 & -1.39473900 & -4.45829900 \\
\hline $\mathrm{O}$ & 0.22623300 & 0.87810700 & -1.69174500 \\
\hline $\mathrm{C}$ & -0.91298300 & 1.21798000 & -2.33412400 \\
\hline $\mathrm{C}$ & -1.49753500 & 0.29689800 & -3.22203800 \\
\hline $\mathrm{C}$ & -2.66975500 & 0.66911000 & -3.88307600 \\
\hline $\mathrm{H}$ & -3.12724700 & -0.03231100 & -4.57671800 \\
\hline $\mathrm{C}$ & -3.25025300 & 1.91389200 & -3.67010400 \\
\hline $\mathrm{H}$ & -4.15907400 & 2.18956400 & -4.19582300 \\
\hline $\mathrm{C}$ & -2.66041300 & 2.80470600 & -2.77794100 \\
\hline $\mathrm{H}$ & -3.11710300 & 3.77564200 & -2.60215400 \\
\hline $\mathrm{C}$ & -1.49218800 & 2.47659200 & -2.09322700 \\
\hline $\mathrm{C}$ & -0.88664200 & 3.42968700 & -1.07799700 \\
\hline $\mathrm{H}$ & -1.06787800 & 4.45668500 & -1.41335400 \\
\hline $\mathrm{H}$ & 0.19585500 & 3.28139100 & -1.03664300 \\
\hline $\mathrm{O}$ & -1.84769200 & 0.00779400 & -0.00586400 \\
\hline $\mathrm{H}$ & -2.26125100 & 0.04160800 & -0.88514800 \\
\hline $\mathrm{H}$ & -2.27913600 & -0.70110200 & 0.49992400 \\
\hline $\mathrm{C}$ & 3.59236800 & -0.00433000 & 0.01192900 \\
\hline $\mathrm{C}$ & 4.27177700 & -1.22942100 & 0.11123000 \\
\hline $\mathrm{C}$ & 4.27389300 & 1.22074500 & -0.08403800 \\
\hline $\mathrm{C}$ & 5.66304400 & -1.20259000 & 0.11045600 \\
\hline $\mathrm{C}$ & 5.66434000 & 1.19209800 & -0.08039700 \\
\hline $\mathrm{C}$ & 6.37562000 & -0.00629000 & $0.012745 c$ \\
\hline
\end{tabular}




$\begin{array}{crrr}\mathrm{H} & 6.20550200 & -2.14231300 & 0.18979100 \\ \mathrm{H} & 6.20856200 & 2.13154700 & -0.15202500 \\ \mathrm{C} & 3.49549500 & 2.50426500 & -0.19215700 \\ \mathrm{H} & 2.90024700 & 2.51978700 & -1.11278300 \\ \mathrm{H} & 2.79021000 & 2.61174500 & 0.63865200 \\ \mathrm{H} & 4.16488600 & 3.36902600 & -0.19898800 \\ \mathrm{C} & 3.49229100 & -2.51161100 & 0.22702500 \\ \mathrm{H} & 2.89769400 & -2.52165700 & 1.14815400 \\ \mathrm{H} & 2.78641300 & -2.62255900 & -0.60284300 \\ \mathrm{H} & 4.16091400 & -3.37695800 & 0.23785000 \\ \mathrm{C} & 7.88310900 & -0.00691200 & -0.02072500 \\ \mathrm{H} & 8.29404700 & -0.89248000 & 0.47366100 \\ \mathrm{H} & 8.25083900 & -0.00623700 & -1.05428300 \\ \mathrm{H} & 8.29365400 & 0.87980500 & 0.47270200 \\ \mathrm{~N} & 2.21428800 & -0.00226900 & 0.01265700\end{array}$

\section{Compound 4}

$\mathrm{E}=-2480.586549$ Hartree

$\begin{array}{lrrr}\text { W } & 1.86167300 & 0.01613700 & -0.00828200 \\ \mathrm{O} & 1.46111000 & -1.17831400 & -1.44038900 \\ \mathrm{C} & 0.30627400 & -1.65502700 & -1.98678700 \\ \mathrm{C} & -0.24824800 & -2.84300800 & -1.50579400 \\ \mathrm{C} & -1.42253500 & -3.31829900 & -2.09073400 \\ \mathrm{H} & -1.84289200 & -4.23612700 & -1.69168300 \\ \mathrm{C} & -2.05417300 & -2.65561700 & -3.14082400 \\ \mathrm{C} & -1.44864800 & -1.48659800 & -3.61178100 \\ \mathrm{H} & -1.89902300 & -0.94178400 & -4.43854400 \\ \mathrm{C} & -0.28141800 & -0.97250100 & -3.05963300 \\ \mathrm{C} & 0.30342600 & 0.33107700 & -3.55906700 \\ \mathrm{H} & 0.09225300 & 0.43124300 & -4.62891600 \\ \mathrm{H} & 1.38936000 & 0.32549400 & -3.43269300 \\ \mathrm{O} & 1.44571900 & 1.44966000 & -1.19499200 \\ \mathrm{C} & 0.28308900 & 1.99746400 & -1.65201100 \\ \mathrm{C} & -0.29638800 & 1.51199400 & -2.82629400 \\ \mathrm{C} & -1.47842800 & 2.09796200 & -3.28052300 \\ \mathrm{H} & -1.91762500 & 1.69650900 & -4.18839600 \\ \mathrm{C} & -2.09447600 & 3.15243500 & -2.61003200 \\ \mathrm{C} & -1.46442900 & 3.62713600 & -1.45586800 \\ \mathrm{H} & -1.90325800 & 4.45574200 & -0.90453500 \\ \mathrm{C} & -0.28797100 & 3.07500500 & -0.96320500 \\ \mathrm{C} & 0.31891400 & 3.57457900 & 0.33030400 \\ \mathrm{H} & 0.11564800 & 4.64580900 & 0.43197100 \\ \mathrm{H} & 1.40394500 & 3.44006100 & 0.31105000 \\ \mathrm{O} & 1.45955200 & 1.20537600 & 1.42808900\end{array}$




\begin{tabular}{|c|c|c|c|}
\hline $\mathrm{C}$ & 0.30163700 & 1.65795300 & 1.98881900 \\
\hline $\mathrm{C}$ & -0.27419200 & 2.84407200 & 1.51567500 \\
\hline $\mathrm{C}$ & -1.44516900 & 3.29629800 & 2.11228100 \\
\hline $\mathrm{H}$ & -1.88578400 & 4.21402500 & 1.72900100 \\
\hline $\mathrm{C}$ & -2.06851100 & 2.61387200 & 3.16116600 \\
\hline $\mathrm{C}$ & -1.44792300 & 1.45419100 & 3.62040300 \\
\hline $\mathrm{H}$ & -1.88144000 & 0.89192700 & 4.44140800 \\
\hline $\mathrm{C}$ & -0.26851300 & 0.96422100 & 3.05848000 \\
\hline $\mathrm{C}$ & 0.34169400 & -0.32779300 & 3.55837500 \\
\hline $\mathrm{H}$ & 0.14344100 & -0.42471600 & 4.63103800 \\
\hline $\mathrm{H}$ & 1.42611200 & -0.30416400 & 3.42073800 \\
\hline $\mathrm{O}$ & 1.46675500 & -1.42057900 & 1.17971900 \\
\hline $\mathrm{C}$ & 0.32571600 & -1.99550400 & 1.65533200 \\
\hline $\mathrm{C}$ & -0.24402400 & -1.52391000 & 2.83967900 \\
\hline $\mathrm{C}$ & -1.40170400 & -2.14011900 & 3.31606100 \\
\hline $\mathrm{H}$ & -1.83394100 & -1.75028400 & 4.23234100 \\
\hline $\mathrm{C}$ & -2.00229000 & -3.21066600 & 2.65671300 \\
\hline $\mathrm{C}$ & -1.38272000 & -3.66861000 & 1.48998100 \\
\hline $\mathrm{H}$ & -1.80993400 & -4.50878200 & 0.94710400 \\
\hline $\mathrm{C}$ & -0.23058200 & -3.08603100 & 0.97539100 \\
\hline $\mathrm{C}$ & 0.36782000 & -3.56793300 & -0.32834700 \\
\hline $\mathrm{H}$ & 1.45080400 & -3.41572500 & -0.32083000 \\
\hline $\mathrm{H}$ & 0.18135500 & -4.64192800 & -0.43326000 \\
\hline $\mathrm{N}$ & 3.56129800 & 0.02577100 & -0.01539000 \\
\hline $\mathrm{C}$ & 4.94339000 & 0.02732900 & -0.01884800 \\
\hline $\mathrm{C}$ & 5.61944300 & 1.25693500 & 0.02223700 \\
\hline $\mathrm{C}$ & 7.01041700 & 1.23127300 & 0.01369100 \\
\hline $\mathrm{C}$ & 7.72446500 & 0.03213100 & -0.03141900 \\
\hline $\mathrm{C}$ & 7.01430200 & -1.16903200 & -0.07485900 \\
\hline $\mathrm{C}$ & 5.62311700 & -1.19965300 & -0.06857500 \\
\hline $\mathrm{C}$ & 4.84484400 & -2.48687000 & -0.11917000 \\
\hline $\mathrm{C}$ & 4.83678200 & 2.54151100 & 0.07153600 \\
\hline $\mathrm{C}$ & 9.23213100 & 0.03461300 & -0.00424100 \\
\hline $\mathrm{H}$ & 7.55236700 & 2.17424800 & 0.04240600 \\
\hline $\mathrm{H}$ & 7.55899300 & -2.10991900 & -0.11592800 \\
\hline $\mathrm{H}$ & 4.21144700 & -2.52631200 & -1.01295800 \\
\hline $\mathrm{H}$ & 4.17738200 & -2.57712200 & 0.74517400 \\
\hline $\mathrm{H}$ & 5.51573800 & -3.35025800 & -0.13199200 \\
\hline $\mathrm{H}$ & 4.16396100 & 2.62582400 & -0.78924200 \\
\hline $\mathrm{H}$ & 5.50453700 & 3.40742300 & 0.07727500 \\
\hline $\mathrm{H}$ & 4.20842600 & 2.58212900 & 0.96882300 \\
\hline $\mathrm{H}$ & 9.63901300 & 0.92599100 & -0.49195000 \\
\hline $\mathrm{H}$ & 9.64254400 & -0.84607800 & -0.50787900 \\
\hline $\mathrm{H}$ & 9.60435700 & 0.02636400 & 1.02764900 \\
\hline
\end{tabular}




\begin{tabular}{|c|c|c|c|}
\hline $\mathrm{C}$ & -3.41358800 & 3.78271900 & -3.07062200 \\
\hline $\mathrm{C}$ & -3.94648500 & 3.14154700 & -4.35954700 \\
\hline $\mathrm{H}$ & -4.88617400 & 3.62425100 & -4.64936600 \\
\hline $\mathrm{H}$ & -3.24210200 & 3.25825600 & -5.19087000 \\
\hline $\mathrm{H}$ & -4.15015100 & 2.07310700 & -4.22494900 \\
\hline $\mathrm{C}$ & -3.37942600 & 3.14769500 & 3.74957300 \\
\hline $\mathrm{C}$ & -3.94328200 & 2.22353800 & 4.83822400 \\
\hline $\mathrm{H}$ & -4.88599400 & 2.63278000 & 5.21796200 \\
\hline $\mathrm{H}$ & -4.14766100 & 1.21948900 & 4.44911100 \\
\hline $\mathrm{H}$ & -3.25761900 & 2.13255200 & 5.68810700 \\
\hline $\mathrm{C}$ & -3.29586900 & -3.87506700 & 3.14152500 \\
\hline $\mathrm{C}$ & -3.82236200 & -3.24609900 & 4.43908500 \\
\hline $\mathrm{H}$ & -4.74566200 & -3.75029500 & 4.74462100 \\
\hline $\mathrm{H}$ & -3.10176200 & -3.34615900 & 5.25856200 \\
\hline $\mathrm{H}$ & -4.05244700 & -2.18254000 & 4.30851700 \\
\hline $\mathrm{C}$ & -3.36400600 & -3.14133600 & -3.77170000 \\
\hline $\mathrm{C}$ & -3.87494800 & -4.43774900 & -3.12768200 \\
\hline $\mathrm{H}$ & -4.80976600 & -4.74465200 & -3.60931400 \\
\hline $\mathrm{H}$ & -3.15681600 & -5.25740700 & -3.24312400 \\
\hline $\mathrm{H}$ & -4.08007200 & -4.30443700 & -2.05933600 \\
\hline $\mathrm{C}$ & -3.20476400 & 5.28636900 & -3.33148300 \\
\hline $\mathrm{H}$ & -2.44781900 & 5.44454200 & -4.10754400 \\
\hline $\mathrm{H}$ & -2.87539600 & 5.81284500 & -2.42977800 \\
\hline $\mathrm{C}$ & -4.47289700 & 3.59336600 & -1.96692500 \\
\hline $\mathrm{H}$ & -4.16813900 & 4.06581400 & -1.02718100 \\
\hline $\mathrm{H}$ & -4.63771600 & 2.52896300 & -1.76714900 \\
\hline $\mathrm{C}$ & -3.14789200 & -3.40222500 & -5.27429900 \\
\hline $\mathrm{H}$ & -2.37814500 & -4.16630900 & -5.42896200 \\
\hline $\mathrm{H}$ & -2.83196700 & -2.49641200 & -5.80197100 \\
\hline $\mathrm{C}$ & -4.44339800 & -2.05652100 & -3.58704500 \\
\hline $\mathrm{H}$ & -4.15610000 & -1.11345800 & -4.06394400 \\
\hline $\mathrm{H}$ & -4.61216000 & -1.85606400 & -2.52336800 \\
\hline $\mathrm{C}$ & -3.04375000 & -5.37237000 & 3.40028300 \\
\hline $\mathrm{H}$ & -2.27185100 & -5.50948900 & 4.16553100 \\
\hline $\mathrm{H}$ & -2.71367400 & -5.89106400 & 2.49434200 \\
\hline $\mathrm{C}$ & -4.37882100 & -3.71442600 & 2.05638300 \\
\hline $\mathrm{H}$ & -4.08018000 & -4.18471300 & 1.11349600 \\
\hline $\mathrm{H}$ & -4.57051700 & -2.65467700 & 1.85568000 \\
\hline $\mathrm{C}$ & -3.13508700 & 4.53588200 & 4.37043000 \\
\hline $\mathrm{H}$ & -2.77426600 & 5.25261900 & 3.62509900 \\
\hline $\mathrm{H}$ & -2.38712600 & 4.47814700 & 5.16874300 \\
\hline $\mathrm{C}$ & -4.43154600 & 3.26452100 & 2.62930100 \\
\hline $\mathrm{H}$ & -4.61024100 & 2.29082600 & 2.16039100 \\
\hline $\mathrm{H}$ & -4.11449000 & 3.95965400 & 1.84504600 \\
\hline
\end{tabular}




$\begin{array}{lrrr}\mathrm{H} & -5.31737100 & -4.17963500 & 2.38090900 \\ \mathrm{H} & -3.96248400 & -5.85970900 & 3.74792300 \\ \mathrm{H} & -5.38119300 & 3.63221700 & 3.03618100 \\ \mathrm{H} & -4.06351300 & 4.93444500 & 4.79694600 \\ \mathrm{H} & -4.14089400 & 5.74984000 & -3.66500100 \\ \mathrm{H} & -5.42716900 & 4.03905600 & -2.27250000 \\ \mathrm{H} & -4.07745800 & -3.75219500 & -5.73892000 \\ \mathrm{H} & -5.39167700 & -2.38177600 & -4.03159000\end{array}$

Compound 4 + NDMA

$E=-2745.029247$ Hartree

$\begin{array}{lrrr}\text { W } & 1.91695400 & 0.01151300 & -0.01252100 \\ \mathrm{O} & 1.65285300 & 0.01321200 & -1.93099400 \\ \mathrm{C} & 0.47787700 & 0.04124700 & -2.59821200 \\ \mathrm{C} & -0.17028800 & -1.16624200 & -2.90494700 \\ \mathrm{C} & -1.41146200 & -1.10541000 & -3.52925000 \\ \mathrm{H} & -1.91606600 & -2.04599200 & -3.74258700 \\ \mathrm{C} & -2.01970400 & 0.10419500 & -3.88534000 \\ \mathrm{C} & -1.32034200 & 1.27765300 & -3.60065500 \\ \mathrm{H} & -1.74513300 & 2.24302500 & -3.86033800 \\ \mathrm{C} & -0.08653400 & 1.27299700 & -2.94951700 \\ \mathrm{C} & 0.57984300 & 2.58220700 & -2.55396200 \\ \mathrm{H} & 0.40631700 & 3.31867400 & -3.34673400 \\ \mathrm{H} & 1.65899800 & 2.43032200 & -2.47096600 \\ \mathrm{O} & 1.66052100 & 1.91447400 & -0.01097200 \\ \mathrm{C} & 0.57584800 & 2.71713800 & -0.01971500 \\ \mathrm{C} & 0.04122400 & 3.13804400 & -1.24652000 \\ \mathrm{C} & -1.03686500 & 4.01611100 & -1.22664900 \\ \mathrm{H} & -1.44162300 & 4.34381700 & -2.18239900 \\ \mathrm{C} & -1.61509200 & 4.47613300 & -0.03738400 \\ \mathrm{C} & -1.07287800 & 4.00524300 & 1.15948500 \\ \mathrm{H} & -1.49329900 & 4.31948100 & 2.11020400 \\ \mathrm{C} & 0.00652300 & 3.12002900 & 1.19255900 \\ \mathrm{C} & 0.51156800 & 2.55075400 & 2.50616700 \\ \mathrm{H} & 0.31049300 & 3.27655500 & 3.30232700 \\ \mathrm{H} & 1.59488500 & 2.41222000 & 2.45011400 \\ \mathrm{O} & 1.64815800 & 0.01000600 & 1.90304300 \\ \mathrm{C} & 0.46993900 & 0.00802300 & 2.56626400 \\ \mathrm{C} & -0.13965700 & 1.22756500 & 2.88497800 \\ \mathrm{C} & -1.37121000 & 1.20297400 & 3.53944500 \\ \mathrm{H} & -1.82794400 & 2.15801700 & 3.78189100 \\ \mathrm{C} & -2.00872200 & 0.01159600 & 3.88886700 \\ \mathrm{H} & -1.36240600 & -1.18333000 & 3.55148800 \\ & -1.82529700 & -2.13569400 & 3.80412400\end{array}$




\begin{tabular}{|c|c|c|c|}
\hline $\mathrm{C}$ & -0.13315400 & -1.21522800 & 2.89991600 \\
\hline $\mathrm{C}$ & 0.51954700 & -2.54126600 & 2.52657800 \\
\hline $\mathrm{H}$ & 0.32743200 & -3.26084800 & 3.33045600 \\
\hline $\mathrm{H}$ & 1.60145500 & -2.40098000 & 2.45785800 \\
\hline $\mathrm{O}$ & 1.60112400 & -1.88133900 & -0.01379300 \\
\hline $\mathrm{C}$ & 0.52364400 & -2.69265300 & -0.00405500 \\
\hline $\mathrm{C}$ & -0.00680200 & -3.11466000 & 1.22346700 \\
\hline $\mathrm{C}$ & -1.09363500 & -3.98025900 & 1.20532000 \\
\hline $\mathrm{H}$ & -1.48482700 & -4.32299900 & 2.16148900 \\
\hline $\mathrm{C}$ & -1.69506300 & -4.41378200 & 0.01565200 \\
\hline $\mathrm{C}$ & -1.16492800 & -3.92688700 & -1.17990900 \\
\hline $\mathrm{H}$ & -1.60382500 & -4.21822500 & -2.12952200 \\
\hline $\mathrm{C}$ & -0.06255500 & -3.07108400 & -1.21492600 \\
\hline $\mathrm{C}$ & 0.44849600 & -2.50557900 & -2.52815600 \\
\hline $\mathrm{H}$ & 1.53495200 & -2.39246600 & -2.47568800 \\
\hline $\mathrm{H}$ & 0.22809500 & -3.22353800 & -3.32622600 \\
\hline $\mathrm{O}$ & -0.39834700 & 0.24559500 & -0.05909900 \\
\hline $\mathrm{N}$ & -2.47479500 & -0.19802300 & 0.03049000 \\
\hline $\mathrm{N}$ & -1.27571700 & -0.62106400 & 0.15895100 \\
\hline $\mathrm{C}$ & -3.50671400 & -1.20684400 & 0.17954700 \\
\hline $\mathrm{H}$ & -3.97397600 & -1.39735000 & -0.79123200 \\
\hline $\mathrm{H}$ & -4.25795500 & -0.86291100 & 0.89256800 \\
\hline $\mathrm{H}$ & -3.02611800 & -2.11445200 & 0.54403100 \\
\hline $\mathrm{C}$ & -2.78172900 & 1.18219200 & -0.31312300 \\
\hline $\mathrm{H}$ & -2.37303500 & 1.41538900 & -1.29840500 \\
\hline $\mathrm{H}$ & -2.32673300 & 1.84886100 & 0.42284000 \\
\hline $\mathrm{H}$ & -3.86622600 & 1.29406700 & -0.31334300 \\
\hline $\mathrm{N}$ & 3.62631500 & -0.01412400 & -0.00911000 \\
\hline $\mathrm{C}$ & 5.00315700 & -0.03776500 & -0.00250400 \\
\hline $\mathrm{C}$ & 5.69452200 & 0.81948000 & 0.86871700 \\
\hline $\mathrm{C}$ & 7.08528000 & 0.77385600 & 0.85837600 \\
\hline $\mathrm{C}$ & 7.78678300 & -0.08704700 & 0.01257600 \\
\hline $\mathrm{C}$ & 7.06371800 & -0.92701600 & -0.83800600 \\
\hline $\mathrm{C}$ & 5.67350800 & -0.92162400 & -0.86512800 \\
\hline $\mathrm{C}$ & 4.87827300 & -1.82121200 & -1.77329900 \\
\hline $\mathrm{C}$ & 4.92237600 & 1.74126600 & 1.77451600 \\
\hline $\mathrm{C}$ & 9.29463200 & -0.09208600 & -0.00650300 \\
\hline $\mathrm{H}$ & 7.63760100 & 1.42909900 & 1.52877000 \\
\hline $\mathrm{H}$ & 7.60009700 & -1.60584000 & -1.49802400 \\
\hline $\mathrm{H}$ & 4.23471500 & -1.23416900 & -2.43804600 \\
\hline $\mathrm{H}$ & 4.21713200 & -2.47482100 & -1.19330400 \\
\hline $\mathrm{H}$ & 5.53692900 & -2.44435900 & -2.38489500 \\
\hline $\mathrm{H}$ & 4.27877100 & 2.41045700 & 1.19262900 \\
\hline $\mathrm{H}$ & 5.59627200 & 2.34841600 & 2.38557200 \\
\hline
\end{tabular}




\begin{tabular}{|c|c|c|c|}
\hline $\mathrm{H}$ & 4.26321400 & 1.17254100 & 2.43990100 \\
\hline $\mathrm{H}$ & 9.70790400 & 0.28866000 & 0.93264800 \\
\hline $\mathrm{H}$ & 9.67726700 & 0.54202600 & -0.81578500 \\
\hline $\mathrm{H}$ & 9.68934300 & -1.10065100 & -0.16704300 \\
\hline $\mathrm{C}$ & -2.81675800 & 5.42722700 & -0.09303300 \\
\hline $\mathrm{C}$ & -2.43646400 & 6.69740600 & -0.87711900 \\
\hline $\mathrm{H}$ & -3.28160900 & 7.39560100 & -0.90931600 \\
\hline $\mathrm{H}$ & -1.58958500 & 7.20482200 & -0.40277400 \\
\hline $\mathrm{H}$ & -2.15389400 & 6.46872200 & -1.90976600 \\
\hline $\mathrm{C}$ & -3.34079700 & -0.02625400 & 4.64938000 \\
\hline $\mathrm{C}$ & -3.14585600 & -0.77483800 & 5.98196600 \\
\hline $\mathrm{H}$ & -4.08256400 & -0.79270000 & 6.55215800 \\
\hline $\mathrm{H}$ & -2.83217900 & -1.81144000 & 5.82171500 \\
\hline $\mathrm{H}$ & -2.38033100 & -0.28368300 & 6.59199000 \\
\hline $\mathrm{C}$ & -2.85873400 & -5.41371000 & 0.06533300 \\
\hline $\mathrm{C}$ & -2.33877200 & -6.74652900 & 0.63828400 \\
\hline $\mathrm{H}$ & -3.14255500 & -7.49187500 & 0.67529100 \\
\hline $\mathrm{H}$ & -1.52952600 & -7.14331800 & 0.01594300 \\
\hline $\mathrm{H}$ & -1.94750100 & -6.61922500 & 1.65313500 \\
\hline $\mathrm{C}$ & -3.40486700 & 0.09920200 & -4.54293800 \\
\hline $\mathrm{C}$ & -3.36043600 & -0.71496000 & -5.84939100 \\
\hline $\mathrm{H}$ & -4.34388700 & -0.71611300 & -6.33453900 \\
\hline $\mathrm{H}$ & -2.63336300 & -0.28679900 & -6.54772300 \\
\hline $\mathrm{H}$ & -3.07688800 & -1.75674000 & -5.66802400 \\
\hline $\mathrm{C}$ & -3.28839900 & 5.85150000 & 1.30473000 \\
\hline $\mathrm{H}$ & -2.49738300 & 6.37120600 & 1.85672700 \\
\hline $\mathrm{H}$ & -3.61720200 & 4.99231400 & 1.90069700 \\
\hline $\mathrm{C}$ & -3.99029400 & 4.72146600 & -0.80044500 \\
\hline $\mathrm{H}$ & -4.29833800 & 3.83051700 & -0.24052900 \\
\hline $\mathrm{H}$ & -3.72234500 & 4.40688500 & -1.81498700 \\
\hline $\mathrm{C}$ & -3.89764400 & 1.51489400 & -4.87405200 \\
\hline $\mathrm{H}$ & -3.22680100 & 2.02338000 & -5.57504300 \\
\hline $\mathrm{H}$ & -3.98865100 & 2.13360000 & -3.97342500 \\
\hline $\mathrm{C}$ & -4.42048900 & -0.54142300 & -3.57686000 \\
\hline $\mathrm{H}$ & -4.48736400 & 0.04051500 & -2.64953200 \\
\hline $\mathrm{H}$ & -4.13773100 & -1.56758900 & -3.31635400 \\
\hline $\mathrm{C}$ & -3.45320400 & -5.68234100 & -1.32427800 \\
\hline $\mathrm{H}$ & -2.71834400 & -6.12975900 & -2.00219300 \\
\hline $\mathrm{H}$ & -3.83170700 & -4.76234100 & -1.78549900 \\
\hline $\mathrm{C}$ & -3.99256000 & -4.88501600 & 0.96511700 \\
\hline $\mathrm{H}$ & -4.43694700 & -3.97610100 & 0.54343200 \\
\hline $\mathrm{H}$ & -3.64446700 & -4.65442400 & 1.97708100 \\
\hline $\mathrm{C}$ & -3.87311300 & 1.37910400 & 4.96428900 \\
\hline $\mathrm{H}$ & -4.04948100 & 1.95936300 & 4.05103000 \\
\hline
\end{tabular}




$\begin{array}{lrrr}\mathrm{H} & -3.18232500 & 1.94167400 & 5.60164000 \\ \mathrm{C} & -4.40489600 & -0.75363500 & 3.80736000 \\ \mathrm{H} & -4.07686100 & -1.75469300 & 3.50733600 \\ \mathrm{H} & -4.62504700 & -0.18055400 & 2.89909400 \\ \mathrm{H} & -4.78824800 & -5.63442500 & 1.05162900 \\ \mathrm{H} & -4.29154600 & -6.38264200 & -1.23903700 \\ \mathrm{H} & -5.33960500 & -0.85905900 & 4.37100600 \\ \mathrm{H} & -4.82802900 & 1.30130400 & 5.49595000 \\ \mathrm{H} & -4.13817000 & 6.53744400 & 1.21611300 \\ \mathrm{H} & -4.85526100 & 5.39171800 & -0.87404200 \\ \mathrm{H} & -4.88760100 & 1.46183100 & -5.34039200 \\ \mathrm{H} & -5.41858900 & -0.57372400 & -4.02990200\end{array}$

Compound $4+\mathrm{H}_{2} \mathrm{O}$

$\mathrm{E}=-2557.023210$ Hartree

$\begin{array}{lrrr}\text { W } & -1.82708400 & 0.01648300 & -0.00448600 \\ \mathrm{O} & -1.53695100 & 0.54754600 & -1.84240600 \\ \mathrm{C} & -0.38233500 & 0.73905700 & -2.52123400 \\ \mathrm{C} & 0.22821200 & 2.00449900 & -2.51601200 \\ \mathrm{C} & 1.43433600 & 2.15917900 & -3.18790000 \\ \mathrm{H} & 1.90141400 & 3.14113400 & -3.16527800 \\ \mathrm{C} & 2.07101900 & 1.10614500 & -3.85513400 \\ \mathrm{C} & 1.42840500 & -0.12975300 & -3.84931800 \\ \mathrm{H} & 1.87597200 & -0.98013600 & -4.35435000 \\ \mathrm{C} & 0.20889000 & -0.33625000 & -3.19728400 \\ \mathrm{C} & -0.42071200 & -1.71763900 & -3.15420300 \\ \mathrm{H} & -0.23060300 & -2.22327400 & -4.10704200 \\ \mathrm{H} & -1.50243300 & -1.62343500 & -3.03337700 \\ \mathrm{O} & -1.56452900 & -1.82571500 & -0.54298800 \\ \mathrm{C} & -0.43528100 & -2.53961300 & -0.75216600 \\ \mathrm{C} & 0.15377900 & -2.55427800 & -2.02693700 \\ \mathrm{C} & 1.33115400 & -3.27246200 & -2.20777400 \\ \mathrm{H} & 1.77872800 & -3.26766200 & -3.19927100 \\ \mathrm{C} & 1.95789800 & -3.97067900 & -1.17054400 \\ \mathrm{C} & 1.33696400 & -3.94188900 & 0.07696700 \\ \mathrm{H} & 1.77973000 & -4.46673100 & 0.91797000 \\ \mathrm{C} & 0.15257000 & -3.23963800 & 0.30832900 \\ \mathrm{C} & -0.42910300 & -3.15048500 & 1.70662900 \\ \mathrm{H} & -0.24200100 & -4.09352900 & 2.23148200 \\ \mathrm{H} & -1.51092900 & -3.00728700 & 1.64771900 \\ \mathrm{O} & -1.54557000 & -0.53414700 & 1.82892400 \\ \mathrm{C} & -0.39569400 & -0.74387700 & 2.51055100 \\ \mathrm{C} & 0.19421600 & -2.01461700 & 2.49841200 \\ \mathrm{C} & 1.40711100 & -2.19793600 & 3.16941500 \\ & & & \\ & & & \\ & & \end{array}$




$\begin{array}{lrrr}\mathrm{H} & 1.85364800 & -3.18673300 & 3.13508000 \\ \mathrm{C} & 2.04440000 & -1.16446400 & 3.85247400 \\ \mathrm{C} & 1.40971900 & 0.08311600 & 3.85829700 \\ \mathrm{H} & 1.87306200 & 0.91866300 & 4.37837900 \\ \mathrm{C} & 0.21044100 & 0.32084800 & 3.19843600 \\ \mathrm{C} & -0.38155600 & 1.71640000 & 3.14190900 \\ \mathrm{H} & -0.18143100 & 2.22342700 & 4.09213400 \\ \mathrm{H} & -1.46549800 & 1.65183500 & 3.01934100 \\ \mathrm{O} & -1.50939200 & 1.83210700 & 0.52818500 \\ \mathrm{C} & -0.36139100 & 2.52422600 & 0.73690000 \\ \mathrm{C} & 0.21909700 & 2.53108500 & 2.01173200 \\ \mathrm{C} & 1.40567900 & 3.23138700 & 2.19422900 \\ \mathrm{H} & 1.85284500 & 3.22105200 & 3.18610800 \\ \mathrm{C} & 2.04238800 & 3.91985200 & 1.15660900 \\ \mathrm{C} & 1.42016800 & 3.90473000 & -0.09040500 \\ \mathrm{H} & 1.86932700 & 4.42680700 & -0.92994800 \\ \mathrm{C} & 0.22741700 & 3.21931700 & -0.32156600 \\ \mathrm{C} & -0.36520400 & 3.14859100 & -1.71641900 \\ \mathrm{H} & -1.44997400 & 3.03057700 & -1.65242700 \\ \mathrm{H} & -9.16044600 & 4.08943200 & -2.23898900 \\ \mathrm{O} & -9.57917900 & -0.33842800 & -0.96087100 \\ \mathrm{~N} & -5.52719900 & -0.00692200 & -0.00715700 \\ \mathrm{C} & -3.53441700 & 0.03367300 & -0.00193100 \\ \mathrm{C} & -4.91215600 & 0.03821800 & 0.00225000 \\ \mathrm{C} & -5.59521900 & -1.11439000 & 0.42490000 \\ \mathrm{C} & -6.98565800 & -1.08174000 & 0.42457500 \\ \mathrm{C} & -7.69529700 & 0.05022900 & 0.01763900 \\ \mathrm{C} & -6.98083300 & 1.17740100 & -0.39276800 \\ \mathrm{H} & -5.58978700 & 1.19720600 & -0.41033300 \\ \mathrm{H} & -4.80600000 & 2.40431000 & -0.85114400 \\ \mathrm{H} & -4.81716600 & -2.32480500 & 0.86691100 \\ \mathrm{H} & -9.20302200 & 0.04329200 & -0.00358300 \\ \mathrm{H} & -7.53145400 & -1.96424300 & 0.75195700 \\ \mathrm{H} & -7.52232300 & 2.06741800 & -0.70648700 \\ \mathrm{H} & -4.17577200 & 2.16414100 & -1.71523500 \\ \mathrm{H} & -4.13308300 & 2.74890800 & -0.05855500 \\ \mathrm{H} & -5.786877000 & -2.49958800\end{array}$




\begin{tabular}{|c|c|c|c|}
\hline $\mathrm{H}$ & 4.01435300 & -6.30782100 & -2.70511000 \\
\hline $\mathrm{H}$ & 2.33697700 & -6.52539500 & -2.16087400 \\
\hline $\mathrm{H}$ & 2.71021900 & -5.36290100 & -3.44231000 \\
\hline $\mathrm{C}$ & 3.38991200 & -1.33859600 & 4.56580900 \\
\hline $\mathrm{C}$ & 3.20705400 & -1.07536900 & 6.07235800 \\
\hline $\mathrm{H}$ & 4.16216200 & -1.18807100 & 6.59916400 \\
\hline $\mathrm{H}$ & 2.83603700 & -0.06278300 & 6.26235900 \\
\hline $\mathrm{H}$ & 2.49003300 & -1.78185700 & 6.50468500 \\
\hline $\mathrm{C}$ & 3.37776000 & 4.62651900 & 1.41701100 \\
\hline $\mathrm{C}$ & 3.18447500 & 5.72078300 & 2.48356800 \\
\hline $\mathrm{H}$ & 4.13467300 & 6.22871100 & 2.68822500 \\
\hline $\mathrm{H}$ & 2.46012300 & 6.46913700 & 2.14401800 \\
\hline $\mathrm{H}$ & 2.81690500 & 5.30331800 & 3.42686100 \\
\hline $\mathrm{C}$ & 3.42704100 & 1.34046900 & -4.53059900 \\
\hline $\mathrm{C}$ & 3.27333600 & 2.40530000 & -5.63283300 \\
\hline $\mathrm{H}$ & 4.23637600 & 2.58917600 & -6.12388600 \\
\hline $\mathrm{H}$ & 2.55678400 & 2.07557700 & -6.39290500 \\
\hline $\mathrm{H}$ & 2.91668000 & 3.35755500 & -5.22661300 \\
\hline $\mathrm{C}$ & 3.83728700 & -5.36291200 & -0.16440300 \\
\hline $\mathrm{H}$ & 3.15242500 & -6.12329300 & 0.22729600 \\
\hline $\mathrm{H}$ & 4.02883000 & -4.62897400 & 0.62703100 \\
\hline $\mathrm{C}$ & 4.32628500 & -3.68082800 & -1.93448900 \\
\hline $\mathrm{H}$ & 4.48828100 & -2.89178400 & -1.19135900 \\
\hline $\mathrm{H}$ & 4.01163200 & -3.20293700 & -2.86830800 \\
\hline $\mathrm{C}$ & 3.98569900 & 0.06067900 & -5.16779800 \\
\hline $\mathrm{H}$ & 3.32388100 & -0.32413000 & -5.95178400 \\
\hline $\mathrm{H}$ & 4.13622100 & -0.72901300 & -4.42239900 \\
\hline $\mathrm{C}$ & 4.43995400 & 1.83240600 & -3.47783300 \\
\hline $\mathrm{H}$ & 4.56487200 & 1.08837400 & -2.68301000 \\
\hline $\mathrm{H}$ & 4.11971300 & 2.76989100 & -3.01132800 \\
\hline $\mathrm{C}$ & 3.94413800 & 5.28160400 & 0.14965200 \\
\hline $\mathrm{H}$ & 3.27160200 & 6.05223700 & -0.24346400 \\
\hline $\mathrm{H}$ & 4.12369000 & 4.54267400 & -0.63993900 \\
\hline $\mathrm{C}$ & 4.40633100 & 3.59479600 & 1.92102300 \\
\hline $\mathrm{H}$ & 4.55271700 & 2.80090500 & 1.18008100 \\
\hline $\mathrm{H}$ & 4.08453600 & 3.12386300 & 2.85573900 \\
\hline $\mathrm{C}$ & 3.96248600 & -2.75184700 & 4.38766800 \\
\hline $\mathrm{H}$ & 4.12915500 & -2.98942800 & 3.33047300 \\
\hline $\mathrm{H}$ & 3.30147700 & -3.51491400 & 4.81358000 \\
\hline $\mathrm{C}$ & 4.40741300 & -0.33516200 & 3.98804300 \\
\hline $\mathrm{H}$ & 4.08568500 & 0.70148200 & 4.13125700 \\
\hline $\mathrm{H}$ & 4.54571800 & -0.49623800 & 2.91315100 \\
\hline $\mathrm{H}$ & 5.37406800 & 4.07688100 & 2.10498400 \\
\hline $\mathrm{H}$ & 4.90168000 & 5.76274500 & 0.37770300 \\
\hline
\end{tabular}




$\begin{array}{rrrr}\mathrm{H} & 5.38005800 & -0.45295700 & 4.48034300 \\ \mathrm{H} & 4.92736200 & -2.82672800 & 4.90080500 \\ \mathrm{H} & 4.78698200 & -5.85880300 & -0.39294600 \\ \mathrm{H} & 5.28576400 & -4.17783600 & -2.12058500 \\ \mathrm{H} & 4.95702500 & 0.27066700 & -5.62873000 \\ \mathrm{H} & 5.41818100 & 2.00614300 & -3.94172200 \\ \mathrm{H} & 0.93215700 & -0.61649900 & 0.63326200 \\ \mathrm{H} & 0.93489300 & -0.17262600 & -0.87439900\end{array}$




\section{NMR spectra}

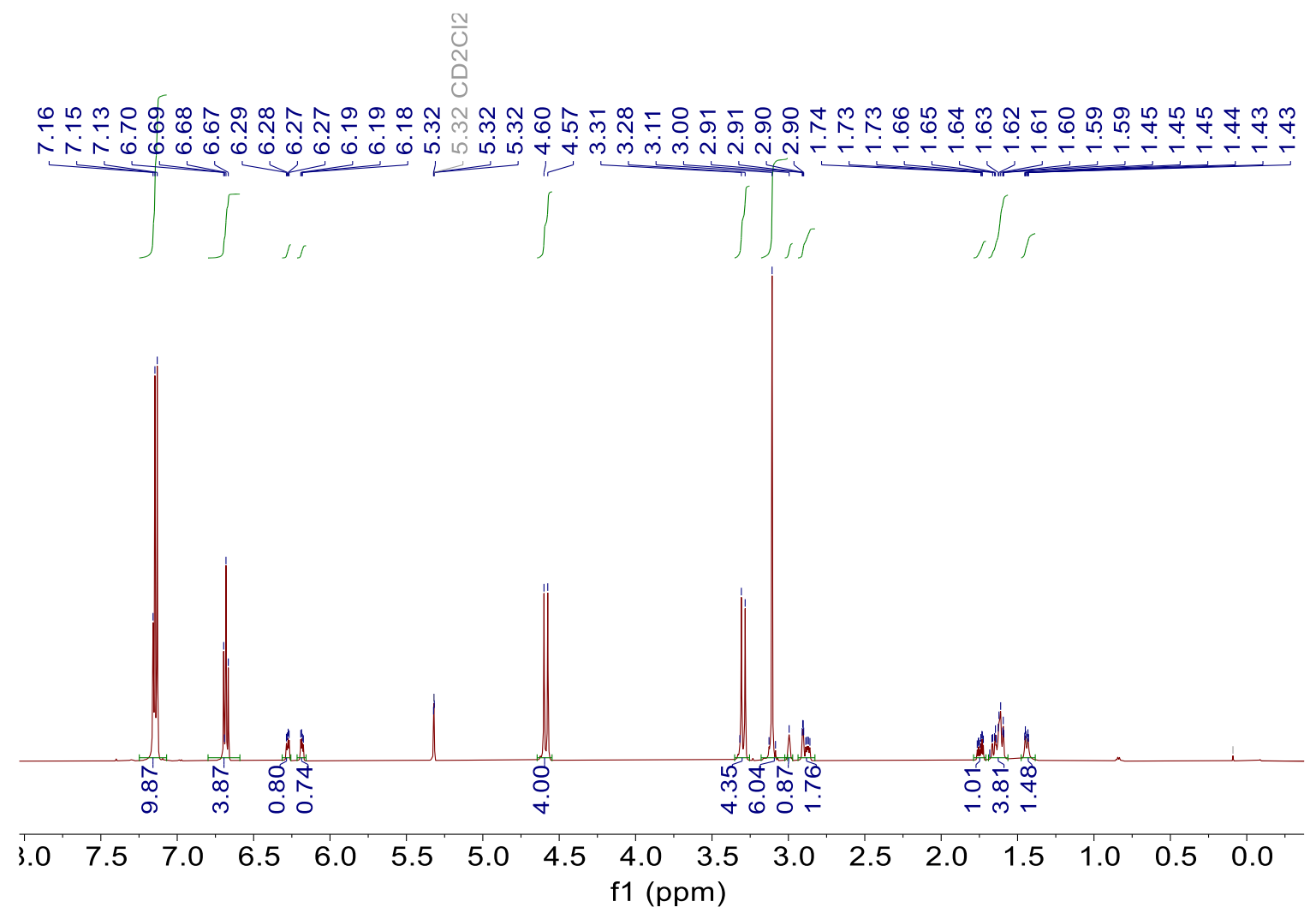

Figure S15. ${ }^{1} \mathrm{H}$ NMR of 1 in $\mathrm{CD}_{2} \mathrm{Cl}_{2}$.

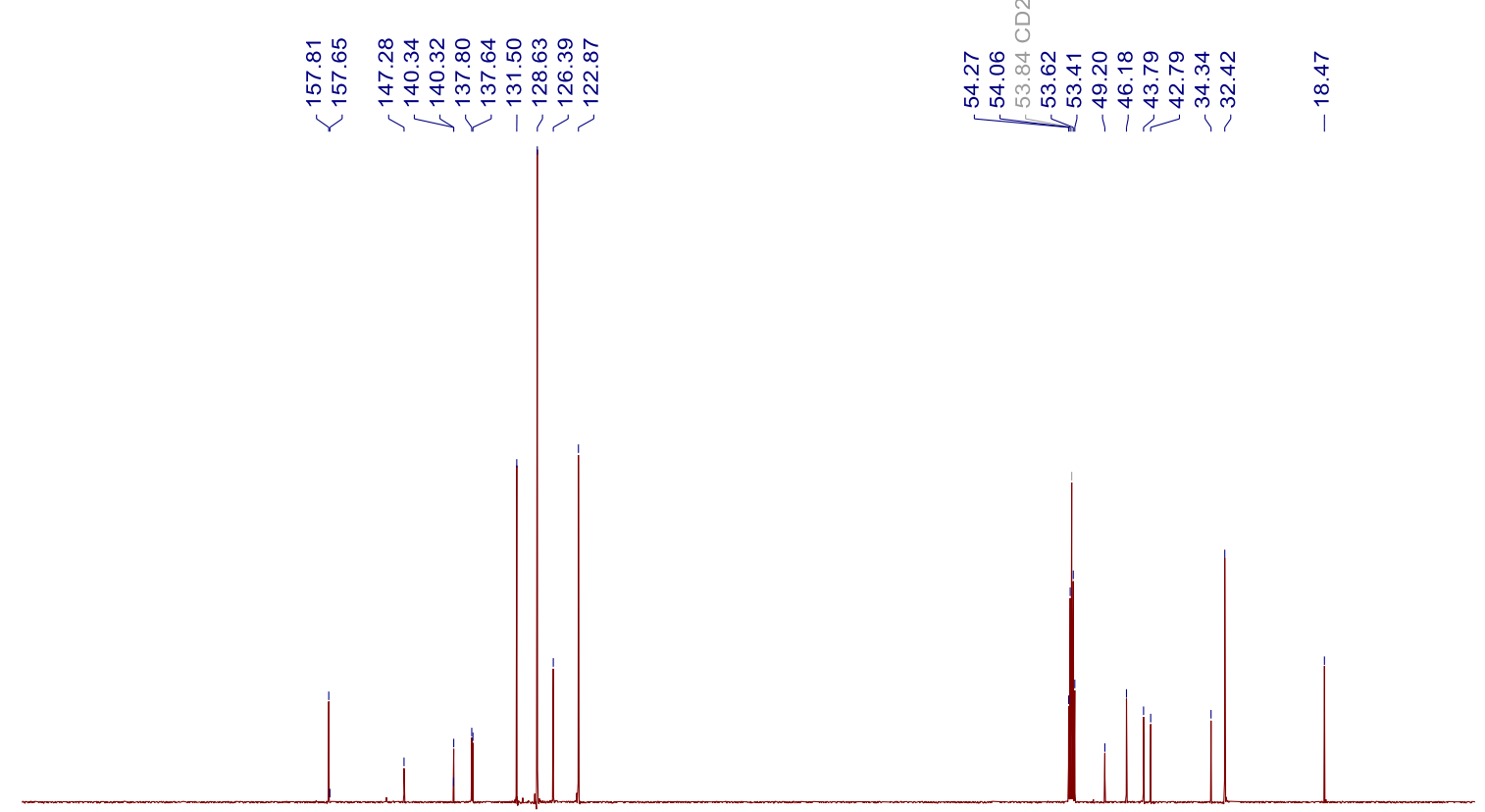

$\begin{array}{lllllllllllllllllllll}00 & 190 & 180 & 170 & 160 & 150 & 140 & 130 & 120 & 110 & 100 & 90 & 80 & 70 & 60 & 50 & 40 & 30 & 20 & 10 & 0\end{array}$ f1 (ppm)

Figure S16. ${ }^{13} \mathrm{C}$ NMR of $\mathbf{1}$ in $\mathrm{CD}_{2} \mathrm{Cl}_{2}$. 

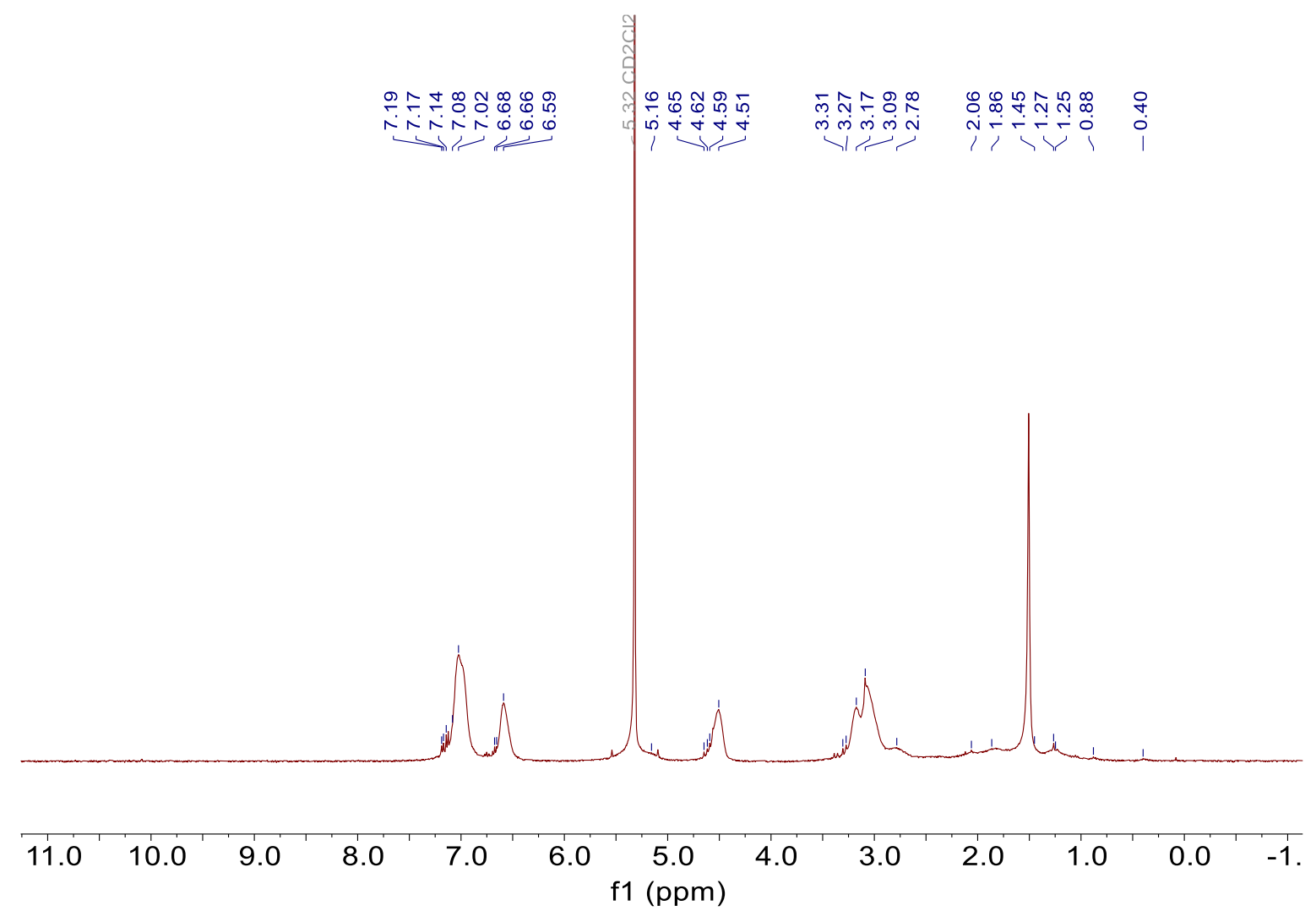

Figure S17. ${ }^{1} \mathrm{H}$ NMR of PCalixH in $\mathrm{CD}_{2} \mathrm{Cl}_{2}$.
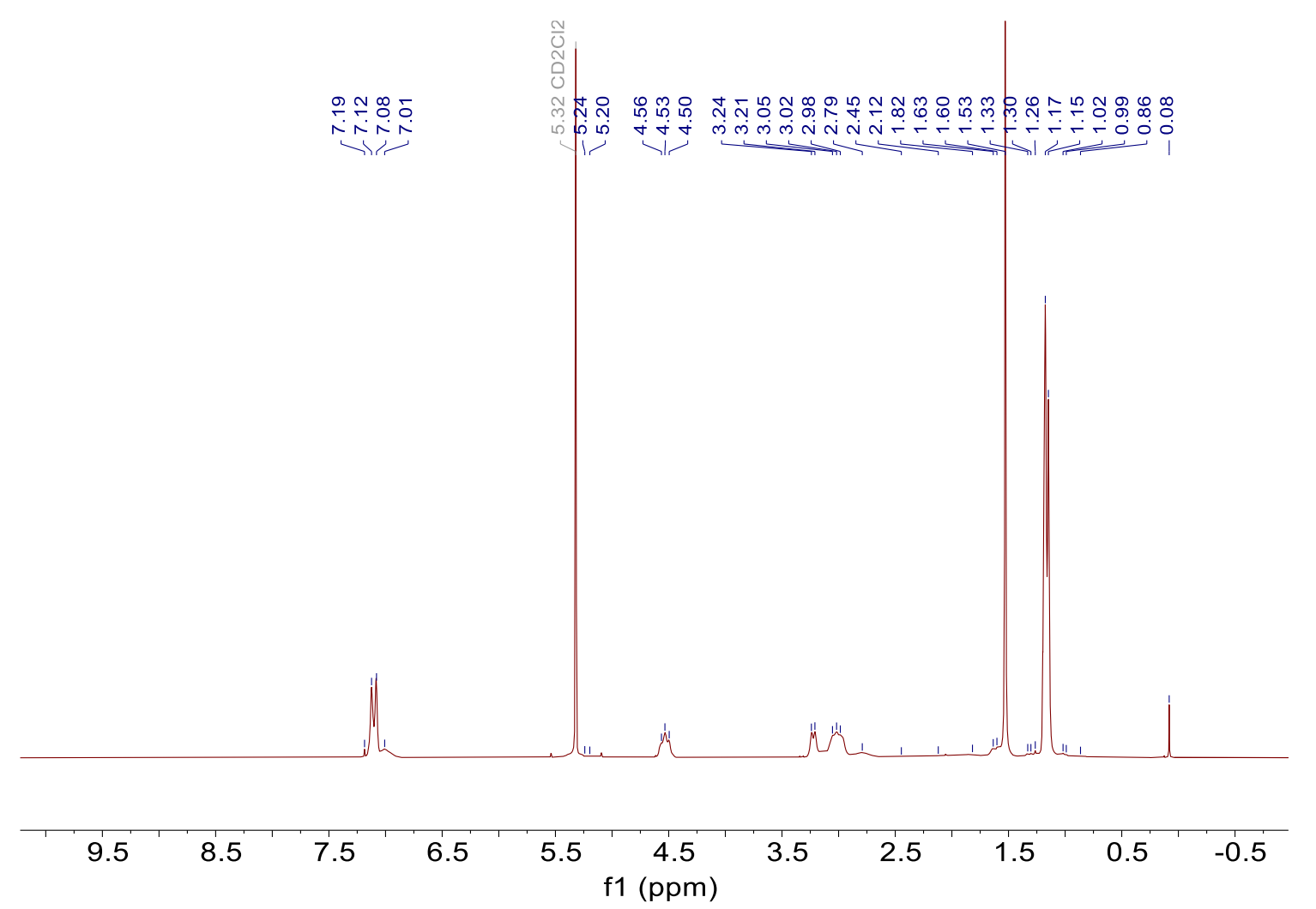

Figure S18. ${ }^{1} \mathrm{H}$ NMR of PCalixtBu in $\mathrm{CD}_{2} \mathrm{Cl}_{2}$. 


\section{Reference}

S1. (a) He, M.; Croy, R. G.; Essigmann, J. M.; Swager, T. M., Chemiresistive Carbon Nanotube Sensors for N-Nitrosodialkylamines. ACS Sens. 2019, 4, 2819-2824; (b) Heath, D. F.; Mattocks, A. R. 823. Preparation of ${ }^{14} \mathrm{C}$-labelled dialkylnitrosamines, and an improved preparation of N-methyl-N-t-butylamine. 1961, 42264229.

S2. Currie, L. A., Nomenclature in evaluation of analytical methods including detection and quantification capabilities (IUPAC Recommendations 1995). Pure Appl. Chem. 1995, 67, 1699-1723.

S3. Li, J.; Lu, Y.; Ye, Q.; Cinke, M.; Han, J.; Meyyappan, M., Carbon Nanotube Sensors for Gas and Organic Vapor Detection. Nano Lett. 2003, 3, 929-933.

S4. Zhao, Y.; Swager, T. M., Functionalized Metalated Cavitands via Imidation and Late-Stage Elaboration. Eur. J. Org. Chem. 2015, 2015, 4593-4597.

S5. Lunn, G.; Sansone, E. B.; Keefer, L. K., Safe disposal of carcinogenic nitrosamines. Carcinogenesis 1983, 4, 315-319.

S6. Chai, J.-D.; Head-Gordon, M., Long-range corrected hybrid density functionals with damped atomatom dispersion corrections. Phys. Chem. Chem. Phys. 2008, 10, 6615-6620.

S7. Gaussian 09, Revision D.01, M. J. Frisch, G. W. Trucks, H. B. Schlegel, G. E. Scuseria, M. A. Robb, J. R. Cheeseman, G. Scalmani, V. Barone, B. Mennucci, G. A. Petersson, H. Nakatsuji, M. Caricato, X. Li, H. P. Hratchian, A. F. Izmaylov, J. Bloino, G. Zheng, J. L. Sonnenberg, M. Hada, M. Ehara, K. Toyota, R. Fukuda, J. Hasegawa, M. Ishida, T. Nakajima, Y. Honda, O. Kitao, H. Nakai, T. Vreven, J. A. Montgomery, Jr., J. E. Peralta, F. Ogliaro, M. Bearpark, J. J. Heyd, E. Brothers, K. N. Kudin, V. N. Staroverov, T. Keith, R. Kobayashi, J. Normand, K. Raghavachari, A. Rendell, J. C. Burant, S. S. Iyengar, J. Tomasi, M. Cossi, N. Rega, J. M. Millam, M. Klene, J. E. Knox, J. B. Cross, V. Bakken, C. Adamo, J. Jaramillo, R. Gomperts, R. E. Stratmann, O. Yazyev, A. J. Austin, R. Cammi, C. Pomelli, J. W. Ochterski, R. L. Martin, K. Morokuma, V. G. Zakrzewski, G. A. Voth, P. Salvador, J. J. Dannenberg, S. Dapprich, A. D. Daniels, O. Farkas, J. B. Foresman, J. V. Ortiz, J. Cioslowski, and D. J. Fox, Gaussian, Inc., Wallingford CT, 2013. 